\title{
Numerical analysis of vehicle-to-vehicle impact using vehicle dynamics control systems for collision mitigation
}

\author{
Ahmed Elmarakbi • Mustafa Elkady • \\ John MacIntyre
}

Received: 9 April 2013 / Revised: 17 May 2013 / Accepted: 28 May 2013 / Published online: 14 June 2013

(C) Springer-Verlag Berlin Heidelberg 2013

\begin{abstract}
This paper focuses on the use of vehicle dynamics control systems (VDCS) to mitigate vehicle collisions in case of offset frontal vehicle-to-vehicle crash scenario. A unique 6-degree-of-freedom vehicle dynamics/crash mathematical model is developed and analyzed in this research. The model is used to define the vehicle body crash parameters by integrating a vehicle dynamics model with a vehicle front-end structure model. In this model, the anti-lock braking system and the active suspension control system are co-simulated with the full car vehicle dynamics model and integrated with the front-end structure. The associated equations of motion of the model are developed and solved numerically. Four different cases of VDCS are investigated in this paper and their associated results are compared with the free rolling case scenario. It is shown from the numerical simulations that the vehicle dynamics/crash response can be captured and analyzed quickly and accurately. Furthermore, it is shown that the VDCS affect the crash characteristics positively.
\end{abstract}

Keywords Vehicle control · Active safety · Collision mitigation · Vehicle dynamics · Mathematical modelling

\footnotetext{
A. Elmarakbi $(\varangle) \cdot$ J. MacIntyre

Department of Computing, Engineering and Technology,

Faculty of Applied Sciences, University of Sunderland,

Sunderland SR6 0DD, UK

e-mail: ahmed.elmarakbi@sunderland.ac.uk

M. Elkady

School of Engineering, Department of Mechanical Engineering,

Lebanese International University (LIU), Mouseitbeh,

Beirut, Lebanon

M. Elkady

Department of Automotive Engineering, Faculty of Engineering,

Ain Shams University, Cairo, Egypt
}

\section{Introduction}

Vehicle dynamics control systems (VDCS) are existed on the most modern vehicles and play important roles in vehicle ride, stability, and safety. For examples, anti-lock braking system (ABS) is used to allow the vehicle to follow the desired steering angle while the intense braking is applied [1] . In addition, the ABS helps reducing the stopping distance of a vehicle compared with the conventional braking system. The active suspension control system (ASC) is used to improve the quality of the vehicle ride and reduce the vertical acceleration $[2,3]$. On the other hand, nowadays, Occupant safety becomes one of the most important research areas and the automotive industry increased their efforts for enhancing the safety of the vehicles. Seat belts, airbags, and advanced driver assistant systems (ADASs) are used to prevent vehicle crash or mitigate vehicle collision when a crash occurs.

Fuzzy control has become one of the most active research fields in the application of the fuzzy set theory. Regarding to the VDCS, fuzzy logic was used to design a controller for the ASC system [4]. Compared to the conventional control theory, fuzzy control does not rely on the analysis of a mathematical model of the process. In addition, its rules are frequently adopted from expert's knowledge or operator's experience [4].

The most well-known pre-collision method is the ADASs. The aim of ADAS is to mitigate and avoid vehicle frontal collisions. The main idea of ADAS is to collect data from the road (i.e. traffic lights, other cars distances and velocities, obstacles etc.) and transfer this information to the driver, warn the driver in danger situations and aide the driver actively in imminent collision. There are different actions may be taken when these systems detect that the collision is unavoidable. For example, the brake assistant system (BAS) [5] and the collision mitigation brake system (CMBS) [6] were used to 
Fig. 1 6-DOF vehicle dynamics/crash mathematical model

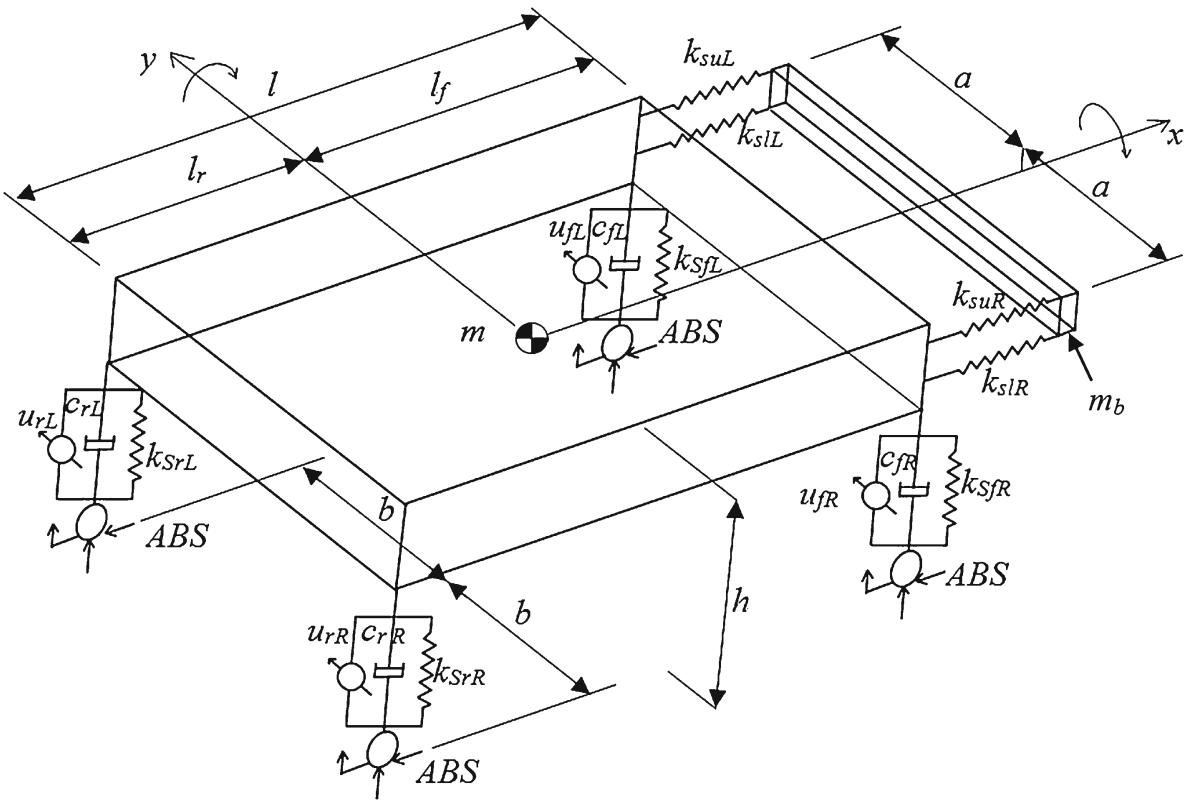

activate the braking instantly based on the behaviour characteristics of the driver.

The vehicle body pitch and drop at fontal impact is the main reason for the unbelted driver neck and head injury [7]. Vehicle pitch and drop are normally experienced at frontal crash tests. Chang et al. [7] used a finite element (FE) method to investigate the frame deformation at full frontal impact and discussed the cause and countermeasures design for the issue of vehicle body pitch and drop. It found that the bending down of frame rails caused by the geometry offsets of the frame rails in vertical direction during crash is the key feature of pitching of the vehicle body.

The effect of vehicle braking on the crash and the possibility of using vehicle dynamics control systems to reduce the risk of incompatibility and improve the crash performance in frontal vehicle-to-barrier collision were investigated [8]. They proved that there is a slight improvement of the vehicle deformation once the brakes are applied during the crash. A multi-body vehicle dynamic model using ADAMS software, alongside with a simple crash model was generated in order to study the effects of the implemented control strategy. The ADAMS multi-body vehicle model including a simple crash structure incorporates a fully independent suspension system. The crash structure of the vehicle consists of four non-linear spring damper sets connecting rigid cross members to the rigid cabin of the vehicle. Their study showed that the control systems were not being able to significantly affect the vehicle crash in the offset barrier impact. In addition, it was found that in offset vehicle-to-vehicle rear-end collision, the ABS or direct yaw control (DYC) systems can stabilize the vehicle; however, these control systems affected each other and cannot work together at the same time.
The main aim of this paper is to investigate the effect of the VDCS on vehicle collisions mitigation and use the VDCS to enhance vehicle crash characteristics in case of offset vehicle-to-vehicle crash scenario.

\section{Integrated vehicle model}

The 6-degree-of-freedom (6-DOF) mathematical model used in this paper, shown in Fig. 1, is developed to optimize the vehicle dynamics control systems (VDCS) in imminent impact in offset crash scenarios. The ABS and the ASC systems are co-simulated with a full car vehicle dynamic model and integrated with a front-end structure. It is worthwhile mentioning that vehicle components, which significantly affect the dynamics of frontal impact, are modeled by lumped masses and nonlinear springs.

In this full-car model, the vehicle body is represented by lumped mass $m$ and it has a translational motion on longitudinal direction (x-axis), translational motion on vertical direction (z-axis), pitching motion (around y-axis), rolling motion (around $\mathrm{x}$-axis), and yawing motion in case of offset collision (around z-axis at the point of impact). In addition, the bumper of the vehicle is represented by a lumped mass $m_{b}$ and it has a rotational motion (around $\mathrm{z}$-axis at the point of impact). Four spring/damper units are used to represent the conventional vehicle suspension systems. Each unit has a spring stiffness $k_{S}$ and a damping coefficient $c$. The subscripts $f, r, R$ and $L$ denote the front, rear, right and left wheels, respectively. The ASC system is co-simulated with the conventional suspension system to add or subtract an active force element $u$. The ABS is co-simulated with the mathematical model using 
Fig. 2 a Vehicle model before crash (offset frontal impact).

b Vehicle model after crash (offset frontal impact)
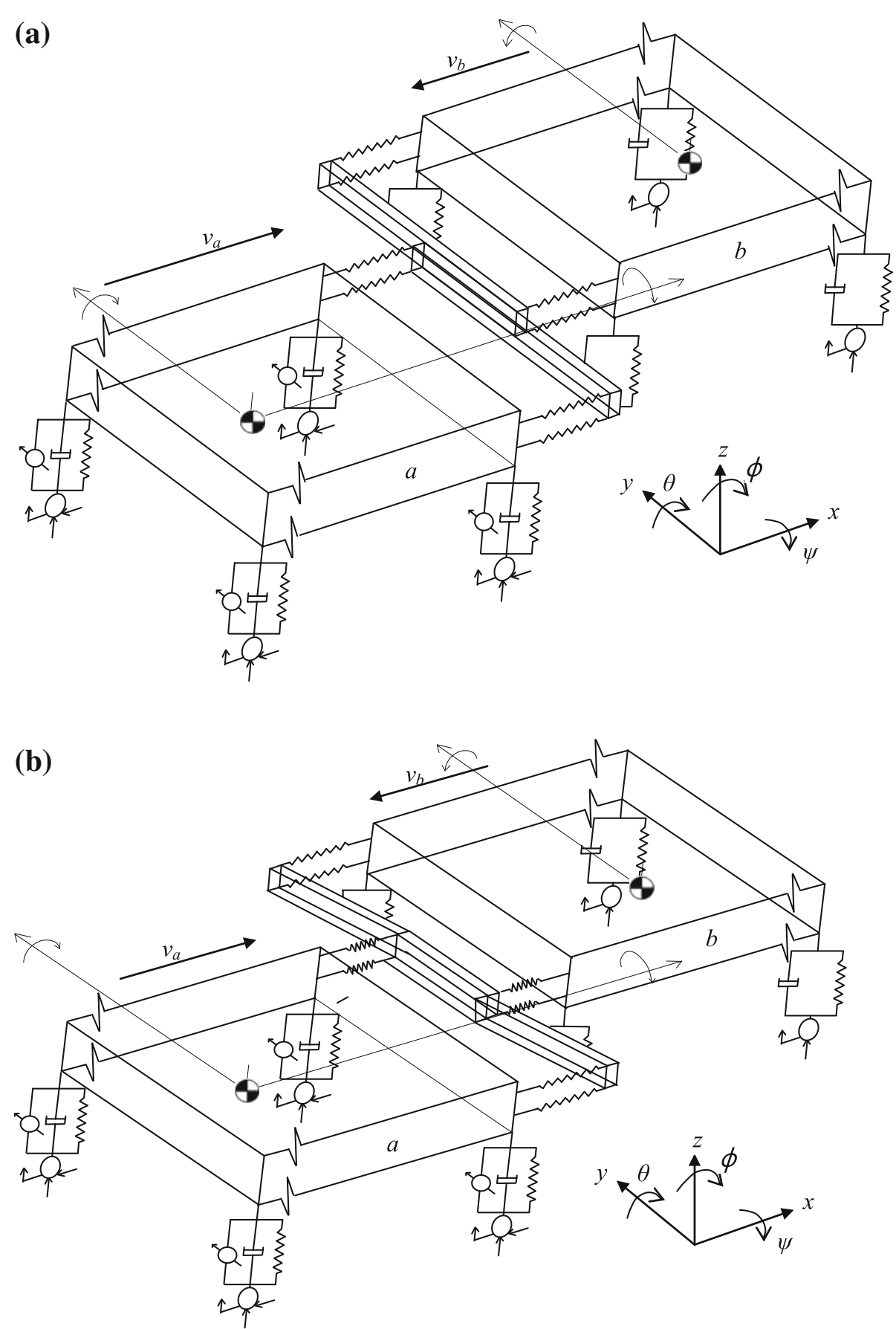

a simple wheel model. The unsprung masses are not considered in this model and it is assumed that the vehicle moves on a flat-asphalted road, which means that the vertical movement of the tires and road vertical forces can be neglected.

To represent the front-end structure of the vehicle, four non-linear springs with stiffness $k_{s}$ are proposed: two springs represent the upper members (rails) and two springs represent the lower members of the vehicle frontal structure. The subscript $u$ denotes the upper rails while the subscript $l$ denotes the lower rails. The general dimensions of the model are shown in Fig. 1, where $l_{f}, l_{r}, l$ and $h$ represent the longitudinal distance between the vehicle's CG and front wheels, the longitudinal distance between the $\mathrm{CG}$ and rear wheels, the wheel base and the high of the $\mathrm{CG}$ from the ground, respectively; $a$ is the distance between the center of the bumper and the right/left frontal springs; $b$ is the distance between the $\mathrm{CG}$ and right/left wheels.

In the offset crash scenario, the vehicle hits another vehicle with a partial contact bumper to bumper as shown in Fig. 2. 

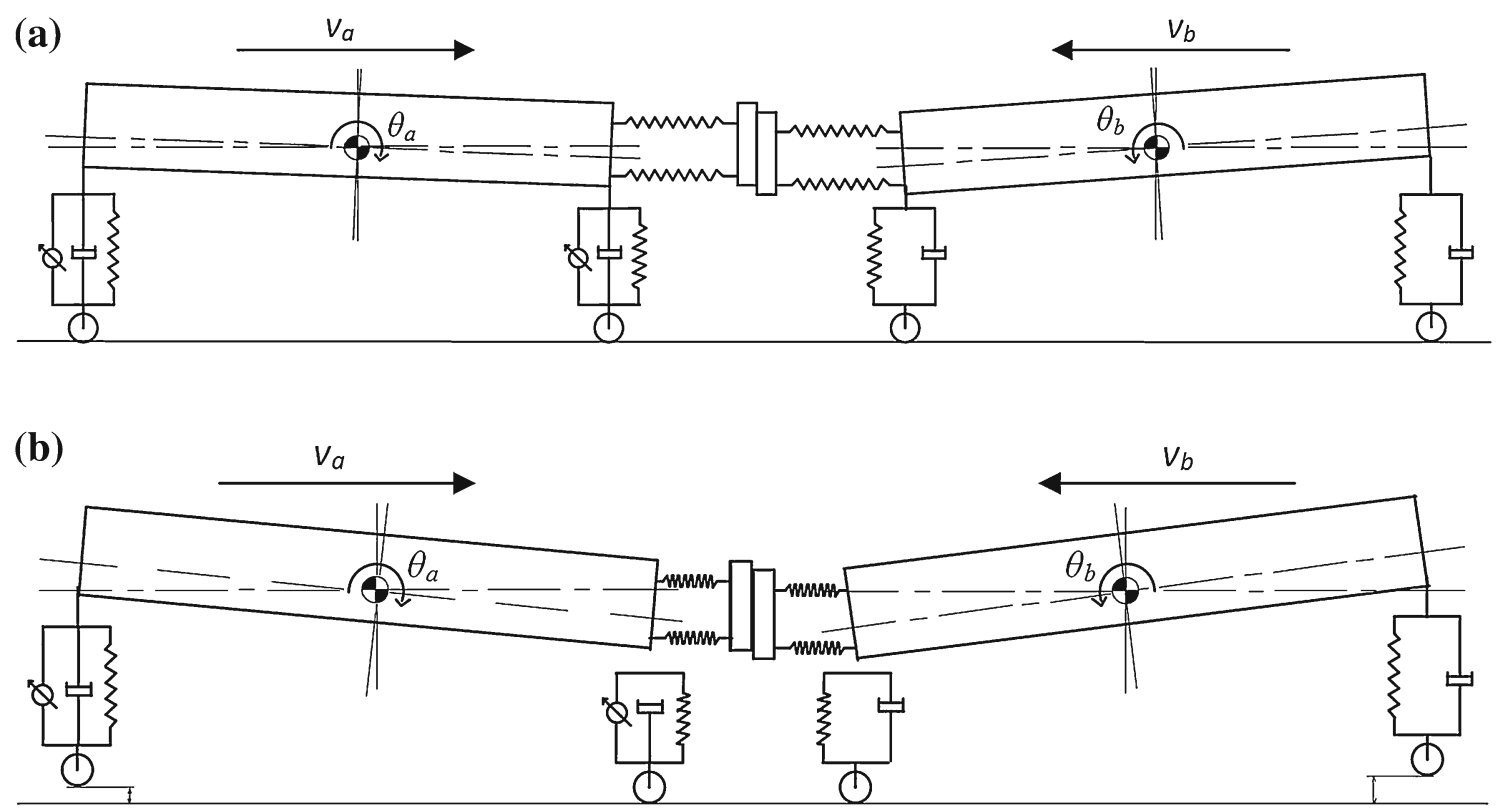

Fig. 3 a The mathematical models at the early stage of the impact. b The mathematical models at the end of impact

Fig. 4 Illustration drawing of the front-end deformation due to vehicle pitching (dashed line before pitching, solid line after pitching)

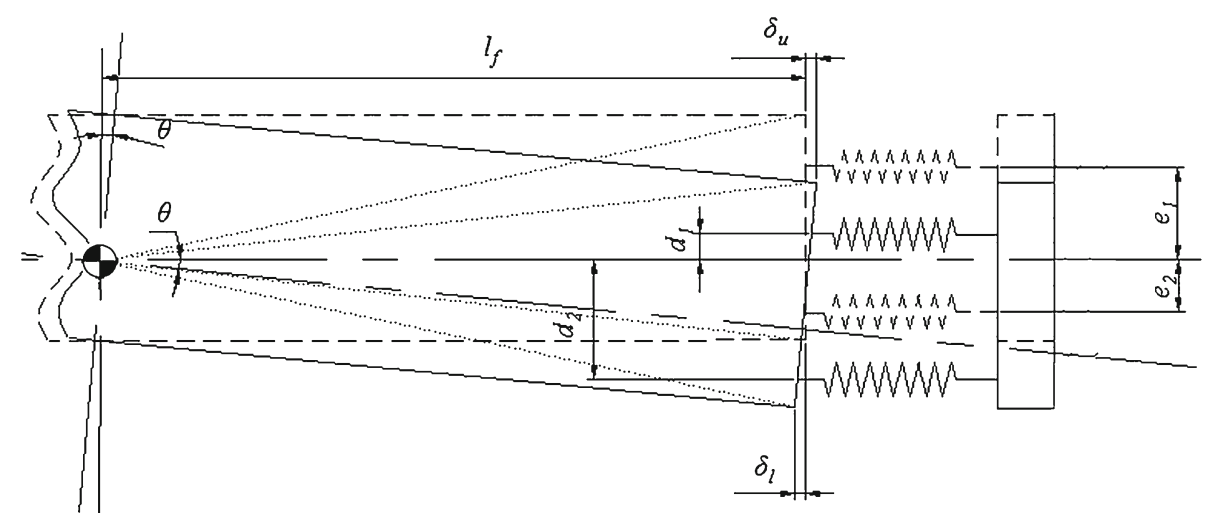

The model in this case (offset frontal vehicle-to-vehicle) is 13-DOF namely longitudinal and vertical movements, pitching, rolling and yawing motions for each vehicle body, the rotational motion for the non-impacted side of each bumper, and the longitudinal movement of the two bumpers as one part. The bumper of each vehicle is considered as two parts of lumped undeformable masses. First part is attached to the impacted side while the second part is attached to the nonimpacted side. First part has only a translation movement and dealt as one mass to transfer the load from one vehicle to another, while the second part has a rotation and translation movements. Figure $2 \mathrm{a}, \mathrm{b}$ shows the half-vehicle model before and after collision (offset frontal vehicle-to-vehicle), respectively.

As shown in Fig. 2, vehicle b is not equipped with any control systems, and conventional braking is not applied during the crash. Figure $3 a, b$ shows the deformation of the front- end and vehicle pitching at the early stage and at the end of impact for the two vehicles, respectively.

The equations of motion of the mathematical model shown in Fig. 2 are developed to study and predict the dynamic response of vehicle-to-vehicle offset frontal crash scenario. Figures 4 and 5 can be used to describe the deformation of the front springs due to vehicle pitching around its $\mathrm{CG}$ and vehicle yawing around the point of impact for the two vehicles, respectively. The equations of motion of the two vehicles models are written as follows:

$$
\begin{aligned}
& m_{a} \cdot \ddot{x}_{a}+\left(F_{s u R a}+F_{s l R a}\right) \cdot \cos \gamma_{a}+\left(F_{s u L a}+F_{s l L a}\right) \cdot \cos \phi_{a} \\
& \quad+F_{b f R a}+F_{b f L a}+F_{b r R a}+F_{b r L a}=0 \\
& m_{b} \cdot \ddot{x}_{b}+\left(F_{s u R b}+F_{s l R b}\right) \cdot \cos \gamma_{b}+\left(F_{s u L b}+F_{s l L b}\right) \cdot \cos \phi_{b} \\
& \quad+F_{b f R b}+F_{b f L b}+F_{b r R b}+F_{b r L b}=0 \\
& m_{a} \cdot \ddot{z}_{a}+F_{S f R a}+F_{S f L a}+F_{S r R a}+F_{S r L a}=0 \\
& m_{a} \cdot \ddot{z}_{b}+F_{S f R b}+F_{S f L b}+F_{S r R b}+F_{S r L b}=0
\end{aligned}
$$


Fig. 5 Illustration drawing of the front-end deformation due to vehicle yawing (dashed line before yawing, solid line after yawing)

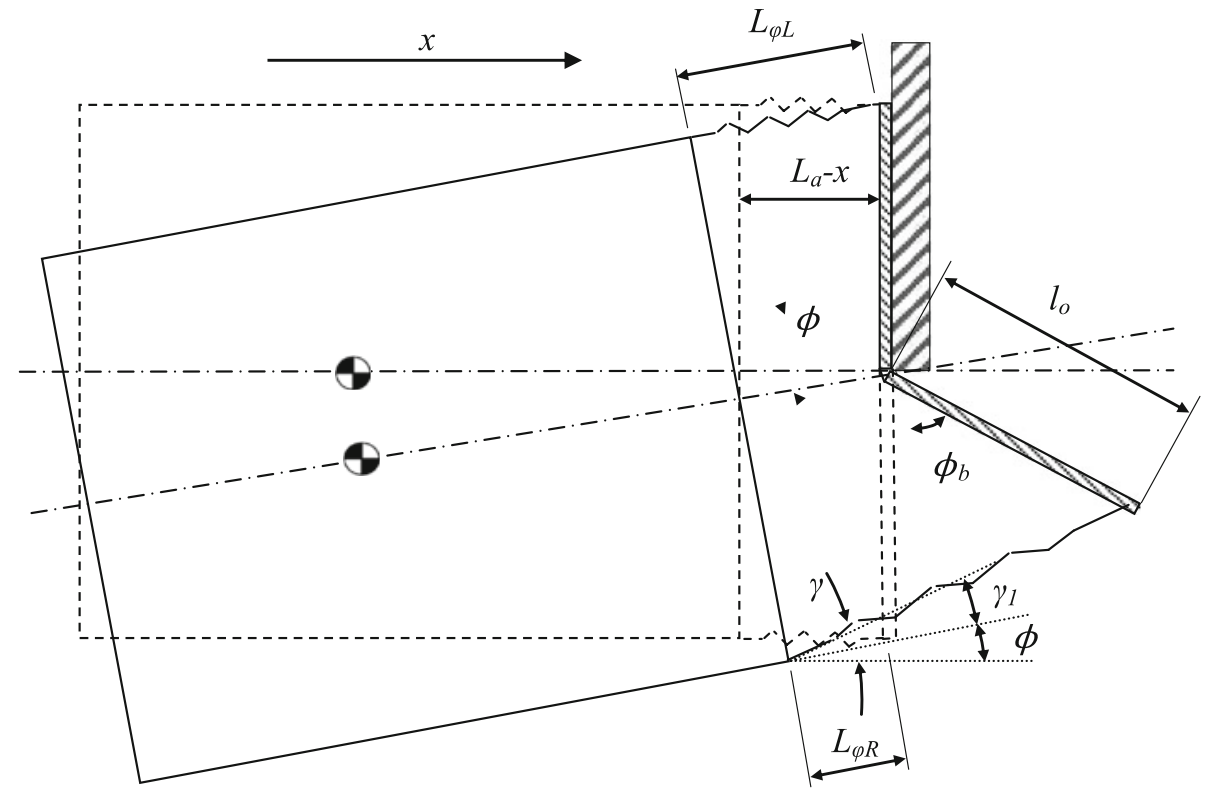

$I_{y y a} \cdot \ddot{\theta}_{a}-\left(F_{S f R a}+F_{S f L a}\right) \cdot l_{f a}+\left(F_{S r R a}+F_{S r L a}\right) \cdot l_{r a}$

$+\left(F_{\text {suRa }} \cdot \cos \gamma_{a}+F_{\text {suLa }} \cdot \cos \phi_{a}\right) \cdot d_{1 a}$

$-\left(F_{s l R a} \cdot \cos \gamma_{a}+F_{s l L a} \cdot \cos \phi_{a}\right) \cdot d_{2 a}$

$-\left(F_{b f R a}+F_{b f L a}+F_{b r R a}+F_{b r L a}\right) \cdot\left(z_{a}+h_{a}\right)=0$

$I_{y y a} \cdot \ddot{\theta}_{b}-\left(F_{S f R b}+F_{S f L b}\right) \cdot l_{f b}+\left(F_{S r R b}+F_{S r L b}\right) \cdot l_{r b}$

$+\left(F_{\text {suRb }} \cdot \cos \gamma_{b}+F_{\text {suLb }} \cdot \cos \phi_{b}\right) \cdot d_{1 b}$

$-\left(F_{s l R b} \cdot \cos \gamma_{b}+F_{s l L b} \cdot \cos \phi_{b}\right) \cdot d_{2 b}$

$-\left(F_{b f R b}+F_{b f L b}+F_{b r R b}+F_{b r L b}\right) \cdot\left(z_{b}+h_{b}\right)=0$

$I_{z z a} \cdot \ddot{\phi}_{a}+\left(F_{\text {suRa }}+F_{\text {slRa }}\right) \cdot \cos \gamma_{1 a} \cdot a_{\text {oa }}$

$-\left(F_{s u L a}+F_{s l L a}\right) \cdot a_{i a}$

$-\left(F_{s u R a}+F_{s l R a}\right) \cdot \sin \gamma_{1 a} \cdot\left(l_{a a}-x_{a}\right)$

$+\left(F_{b f R a}+F_{b r R a}\right) \cdot b_{o a}-\left(F_{b f L a}+F_{b r L a}\right) \cdot b_{i a}$

$+\left(F_{f^{\prime} f R a}+F_{f^{\prime} f L a}\right) \cdot\left(l_{b a}-x_{a}\right)$

$+\left(F_{f^{\prime} r R a}+F_{f^{\prime} r L a}\right) \cdot\left(l_{a}+l_{b a}-x_{a}\right)=0$

$I_{z z b} \cdot \ddot{\phi}_{b}+\left(F_{s u R b}+F_{s l R b}\right) \cdot \cos \gamma_{1 b} \cdot a_{o b}$

$-\left(F_{s u L b}+F_{s l L b}\right) \cdot a_{i b}-\left(F_{s u R b}+F_{s l R b}\right) \cdot \sin \gamma_{1 b} \cdot\left(l_{a b}-x_{b}\right)$

$+\left(F_{b f R b}+F_{b r R b}\right) \cdot b_{o b}-\left(F_{b f L b}+F_{b r L b}\right) \cdot b_{i b}$

$+\left(F_{f^{\prime} f R b}+F_{f^{\prime} f L b}\right) \cdot\left(l_{b b}-x_{b}\right)$

$+\left(F_{f^{\prime} r R b}+F_{f^{\prime} r L b}\right) \cdot\left(l_{b}+l_{b b}-x_{b}\right)=0$

$I_{x x a} \cdot \ddot{\psi}_{a}+\left(F_{S f L a}+F_{S r L a}\right) \cdot b_{i a}-\left(F_{S f R a}+F_{S r R a}\right) \cdot b_{o a}$

$-\left(F_{f^{\prime} f R a}+F_{f^{\prime} f L a}+F_{f^{\prime} r R a}+F_{f^{\prime} r L a}\right) \cdot\left(z_{a}+h_{a}\right)$

$-F_{s u R a} \cdot \sin \gamma_{1 a} \cdot e_{1 a}+F_{s l R a} \cdot \sin \gamma_{1 a} \cdot e_{2 a}=0$

$I_{x x b} \cdot \ddot{\psi}_{b}+\left(F_{S f L b}+F_{S r L b}\right) \cdot b_{i b}-\left(F_{S f R b}+F_{S r R b}\right) \cdot b_{o b}$

$-\left(F_{f^{\prime} f R b}+F_{f^{\prime} f L b}+F_{f^{\prime} r R b}+F_{f^{\prime} r L b}\right) \cdot\left(z_{b}+h_{b}\right)$

$-F_{s u R b} \cdot \sin \gamma_{1 b} \cdot e_{1 b}+F_{s l R b} \cdot \sin \gamma_{1 b} \cdot e_{2 b}=0$

$I_{z z b a} \cdot \ddot{\phi}_{b a}-\left(F_{s u R a}+F_{s l R a}\right) \cdot \cos \gamma_{a} \cdot l_{o a} \cdot \cos \phi_{b a}$

$-\left(F_{s u R a}+F_{s l R a}\right) \cdot \sin \gamma_{a} \cdot l_{o a} \cdot \sin \phi_{b a}=0$

$I_{z z b b} \cdot \ddot{\phi}_{b b}-\left(F_{s u R b}+F_{s l R b}\right) \cdot \cos \gamma_{b} \cdot l_{o b} \cdot \cos \phi_{b b}$

$-\left(F_{s u R b}+F_{s l R b}\right) \cdot \sin \gamma_{b} \cdot l_{o b} \cdot \sin \phi_{b b}=0$

$$
\begin{array}{r}
m_{c} \cdot \ddot{x}_{c}+\left(F_{s u R b}+F_{s l R b}\right) \cdot \cos \gamma_{b}+\left(F_{s u L b}+F_{s l L b}\right) \cdot \cos \phi_{b} \\
-\left(F_{s u R a}+F_{s l R a}\right) \cdot \cos \gamma_{a}-\left(F_{\text {suLa }}+F_{s l L a}\right) \cdot \cos \phi_{a}=0
\end{array}
$$

where $F_{S}, F_{S}, F_{b}, F_{z}$ and $F_{f^{\prime}}$ are front-end non-linear spring forces, vehicle suspension forces, braking forces, normal forces and friction forces between the tires and the road due to vehicle yawing, respectively. Subscript a denotes vehicle $\mathbf{a}$ which is equipped by the VDCS and subscript $\mathbf{b}$ denotes vehicle $\mathbf{b}$ which is used in a free rolling case for all crash scenarios. It is assumed that the two vehicle bumpers are moved in the longitudinal direction of $\mathrm{x}$-axis as one part and subscript $c$ denotes the equivalent of the two bumpers. $d_{1}$ and $d_{2}$ represent the distance between the $\mathrm{CG}$ and the upper springs force and the lower springs force for each vehicle, respectively and can be calculated using Fig. 4 as

$$
\begin{aligned}
& d_{1 a}=\sqrt{l_{f a}^{2}+e_{1 a}^{2}} \cdot \sin \left(\tan ^{-1}\left(\frac{e_{1 a}}{l_{f a}}\right)-\theta_{a}\right) \\
& d_{1 b}=\sqrt{l_{f b}^{2}+e_{1 b}^{2}} \cdot \sin \left(\tan ^{-1}\left(\frac{e_{1 b}}{l_{f a}}\right)-\theta_{b}\right) \\
& d_{2 a}=\sqrt{l_{f a}^{2}+e_{2 a}^{2}} \cdot \sin \left(\tan ^{-1}\left(\frac{e_{2 a}}{l_{f a}}\right)-\theta_{a}\right) \\
& d_{2 b}=\sqrt{l_{f b}^{2}+e_{2 b}^{2}} \cdot \sin \left(\tan ^{-1}\left(\frac{e_{1 b}}{l_{f a}}\right)-\theta_{b}\right)
\end{aligned}
$$

where angles $\gamma$ and $\gamma_{1}$ for each vehicle can also be calculated using Fig. 5 as

$$
\begin{aligned}
& \gamma_{a}=\tan ^{-1}\left(\frac{l_{o a}-l_{o a} \cdot \cos \phi_{b a}}{l_{o a} \cdot \sin \phi_{b a}+l_{\phi R a} \cdot \cos \phi_{a}}\right) \\
& \gamma_{b}=\tan ^{-1}\left(\frac{l_{o b}-l_{o b} \cdot \cos \phi_{b b}}{l_{o b} \cdot \sin \phi_{b b}+l_{\phi R b} \cdot \cos \phi_{b}}\right)
\end{aligned}
$$


Fig. 6 Force deformation characteristics for upper and lower rails

Fig. 7 General piecewise force-deformation characteristics
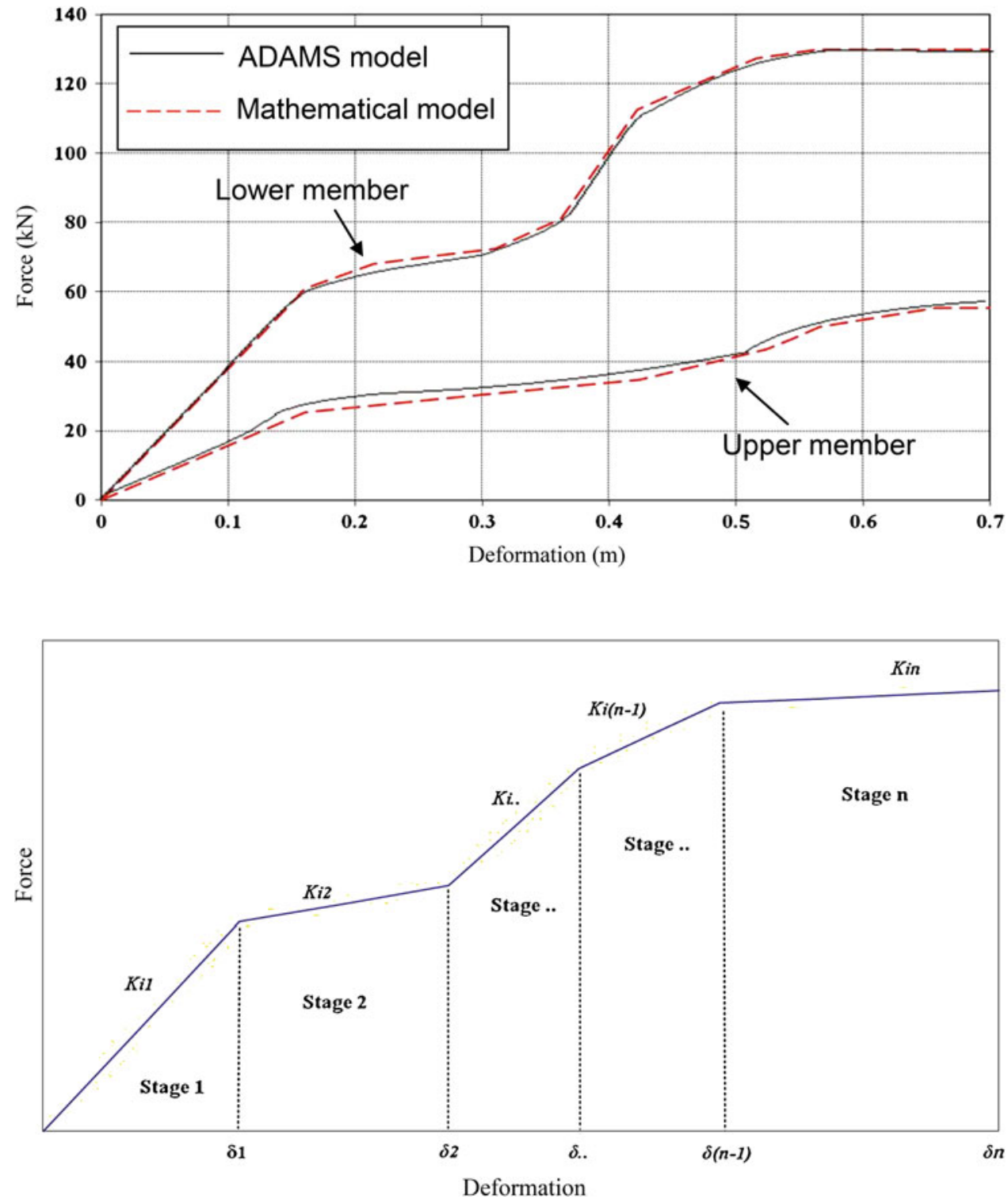

$l_{\phi R a}=\frac{\left(l_{a a}-x_{a}\right)-a_{o a} \cdot \sin \phi_{a}}{\cos \phi_{a}}$

$l_{\phi R b}=\frac{\left(l_{a b}-x_{b}\right)-a_{o b} \cdot \sin \phi_{b}}{\cos \phi_{b}}$

$\gamma_{1 a}=\gamma_{a}-\phi_{a}$

$\gamma_{1 b}=\gamma_{b}-\phi_{b}$

\section{Forces applied on the vehicle model}

There are different types of forces which are applied on the vehicle body. These forces are generated by crushing the front-end structure $F_{S}$, suspension system due to the movement of the vehicle body $F_{S}$, the braking force $F_{b}$, and the friction forces between the tires and the road due to vehicle yawing $F_{f^{\prime}}$.
To simulate the upper and lower members of the vehicle front-end structure, multi-stage piecewise linear forcedeformation spring characteristics are considered. The nonlinear springs used in the multi-body model (ADAMS) [8] are simply extracted to generate the $n$ stage piecewise spring's characteristics as shown in Fig. 6. The forces of the front-end springs are calculated using the general relationship between the force and deflection of a non-linear spring depicted in Fig. 7 as follows:

$F_{s i}=k_{s i j} \delta_{i}+F_{i j}$

where $k_{s}$ and $\delta$ represent the stiffness and the deflection of the front-end spring, respectively. The subscript $i$ indicates the spring location $\left(u_{R}\right.$ : upper right spring, $u_{L}$ : upper left spring, $l_{R}$ : lower right spring and $l_{L}$ : lower left spring) and the subscript $j$ indicates different stages of the force-deformation 
characteristics as shown in Fig. 7. The stiffness of the spring $k_{s}$ and the force elements $F_{i j}$ vary according to the different stages of the deflection $\delta$ and can be defined as follows:

$$
\begin{aligned}
& k_{s i j}=k_{s i 1}, \quad F_{i j}=0 \quad 0 \leq \delta<\delta_{i 1} \\
& k_{s i j}=k_{s i 2}, \quad F_{i j}=\left(k_{s i 1}-k_{s i 2}\right) \cdot \delta_{i 1} \quad \delta_{i 1} \leq \delta<\delta_{i 2} \\
& k_{s i j}=k_{s i 3}, \quad F_{i j}=\left(k_{s i 1}-k_{s i 2}\right) \cdot \delta_{i 1} \\
& \quad+\left(k_{s i 2}-k_{s i 3}\right) \cdot \delta_{i 2} \quad \delta_{i 2} \leq \delta<\delta_{i 3} \\
& k_{s i j}=k_{\sin }, \quad F_{i j}=\left(k_{s i 1}-k_{s i 2}\right) \cdot \delta_{i 1}+\left(k_{s i 2}-k_{s i 3}\right) \cdot \delta_{i 2} \\
& \quad+\ldots+\left(k_{s i(n-1)}-k_{\sin }\right) \cdot \delta_{i(n-1)} \quad \delta>\delta_{(n-1)}
\end{aligned}
$$

where the deformation of the front-end springs $\delta_{i}$ can be calculated using Figs. 4 and 5 as follows:

$$
\begin{aligned}
\delta_{u R} & =x+\delta_{\theta u R}+\delta_{\phi u R}-\delta_{b} \\
\delta_{u L} & =x+\delta_{\theta u L}+\delta_{\phi u L} \\
\delta_{L R} & =x+\delta_{\theta L R}+\delta_{\phi L R}-\delta_{b} \\
\delta_{l L} & =x-\delta_{\theta l L}+\delta_{\phi l L}
\end{aligned}
$$

where $\delta_{\theta}, \delta_{\varphi}$ and $\delta_{b}$ represent the deflection of the front end due to pitching, yawing and the bumper's rotation, respectively and can be calculated as

$$
\begin{aligned}
& \delta_{\theta u R}=\delta_{\theta u L}=\sqrt{l_{f}^{2}+e_{1}^{2}} \cdot \cos \left(\tan ^{-1}\left(\frac{e_{1}}{l_{f}}\right)-\theta\right)-l_{f} \\
& \delta_{\theta l R}=\delta_{\theta l L}=l_{f}-\left[\sqrt{l_{f}^{2}+e_{2}^{2}} \cdot \cos \left(\tan ^{-1}\left(\frac{e_{2}}{l_{f}}\right)+\theta\right)\right] \\
& \delta_{\phi u R}=\delta_{\phi l R}=\left(l_{a}-x\right)-l_{\phi R} \\
& \delta_{\phi u L}=\delta_{\phi l L}=l_{\phi L}-\left(l_{a}-x\right) \\
& l_{\phi L}=\frac{\left(l_{a}-x\right)+a_{i} \cdot \sin \phi}{\cos \phi} \\
& \delta_{b}=\sqrt{\left(l_{o}-l_{o} \cos \phi_{1}\right)^{2}+\left(l_{o} \sin \phi_{1}+l_{\phi R} \cos \phi\right)^{2}}-l_{\phi R}
\end{aligned}
$$

The suspension forces of the vehicle body can be written as follows:

$$
\begin{aligned}
F_{S f R}= & k_{S f R}\left(z-l_{f} \cdot \sin \theta-b_{o} \cdot \psi\right) \\
& +c_{f R}\left(\dot{z}-l_{f} \cdot \dot{\theta} \cos \theta-b_{o} \cdot \dot{\psi}\right)-u_{f R} \\
F_{S f L}= & k_{S f L}\left(z-l_{f} \cdot \sin \theta+b_{i} \cdot \psi\right) \\
& +c_{f L}\left(\dot{z}-l_{f} \cdot \dot{\theta} \cos \theta+b_{i} \cdot \dot{\psi}\right)-u_{f L} \\
F_{S r R}= & k_{S r R}\left(z+l_{r} \cdot \sin \theta-b_{o} \cdot \psi\right) \\
& +c_{r R}\left(\dot{z}+l_{r} \cdot \dot{\theta} \cos \theta-b_{o} \cdot \dot{\psi}\right)-u_{r R} \\
F_{S r L}= & k_{S r L}\left(z+l_{r} \cdot \sin \theta+b_{i} \cdot \psi\right) \\
& +c_{r L}\left(\dot{z}+l_{r} \cdot \dot{\theta} \cos \theta+b_{i} \cdot \dot{\psi}\right)-u_{r L}
\end{aligned}
$$

where $\theta$ and $\psi$ are the vehicle body pitching and rolling angles, respectively, and $\dot{z}, \dot{\theta}$ and $\dot{\psi}$ are the vehicle body vertical, pitching and rolling velocities, respectively. The ASC force elements $(u)$ are applied in the vertical direction parallel to the existing conventional suspension system.
Table 1 Vehicle dynamics control systems cases

\begin{tabular}{ll}
\hline Case & Description \\
\hline Case 1 & Free rolling \\
Case 2 & ABS \\
Case 3 & ABS + ASC \\
Case 4 & ABS + APC system \\
Case 5 & ABS + UPC system \\
\hline
\end{tabular}

\section{Control systems}

Five analyses cases, as described in Table 1, are investigated in this paper. Four different cases of vehicle dynamics control systems (VDCS) are studies and their associated results are compared with the free rolling case scenario. The different cases include combinations of the following systems: ABS, ASC, anti-pitch control (APC) and under-pitch control (UPC).

\subsection{Free rolling}

In the case of free rolling, the vehicle collides with another vehicle without applying any types of control.

\subsection{Anti-lock braking system control system}

In this case the ABS is applied before and during the collision. The ABS is co-simulated with the mathematical model using PID controller to investigate its effect on vehicle collision. To calculate the braking force $\left(F_{b k}\right)$ generated from the ABS, a simple wheel-road model shown in Fig. 8a is used and its associated equations can be written as

$$
\begin{aligned}
I \dot{\omega} & =T_{w}-T_{b k}=F_{b k} \cdot r_{w}-T_{b k} \\
F_{b k} & =\mu(\lambda) \cdot F_{z k}
\end{aligned}
$$

where the slip ratio $\lambda$ is defined as:

$\lambda=\frac{v-v_{w}}{v}=\frac{v-\omega \cdot r_{w}}{v}$

and $\mathrm{I}$ is the wheel moment of inertia $\left(I_{f}=1.4 \mathrm{~kg} \mathrm{~m}^{2}\right.$ and $I_{r}=1 \mathrm{~kg} \mathrm{~m}^{2}$, related to front and rear wheels respectively), $\omega$ is the wheel angular velocity, $\dot{\omega}$ is the wheel angular acceleration, $T_{w}$ is the friction torque, $T_{b}$ is the braking torque applied by the disk/drum brakes, $r_{w}$ is the wheel radius (has been taken as $0.33 \mathrm{~m}), \mu$ is the friction coefficient between the tire and the road, $\lambda$ is the tire slip ratio, $F_{z}$ is the vertical normal forces of the tires, $v$ is the vehicle body velocity, and $v_{w}$ is the equivalent wheel longitudinal velocity. The relation between $\mu$ and $\lambda$ is shown in Fig. $8 \mathrm{~b}$. The subscript $\mathrm{k}$ indicates the wheel's location $\left(f_{R}\right.$ : front right wheel, $f_{L}$ : front left wheel, $r_{R}$ : rear right wheel and $r_{L}$ : rear left wheel). The wheel rotational angle can be calculated by the integration of 
Fig. 8 a Wheel-road model [9]; $\mathbf{b}$ the relationship between friction coefficient and wheel slip [10]

Fig. 9 schematic diagram of the ASC fuzzy logic controller
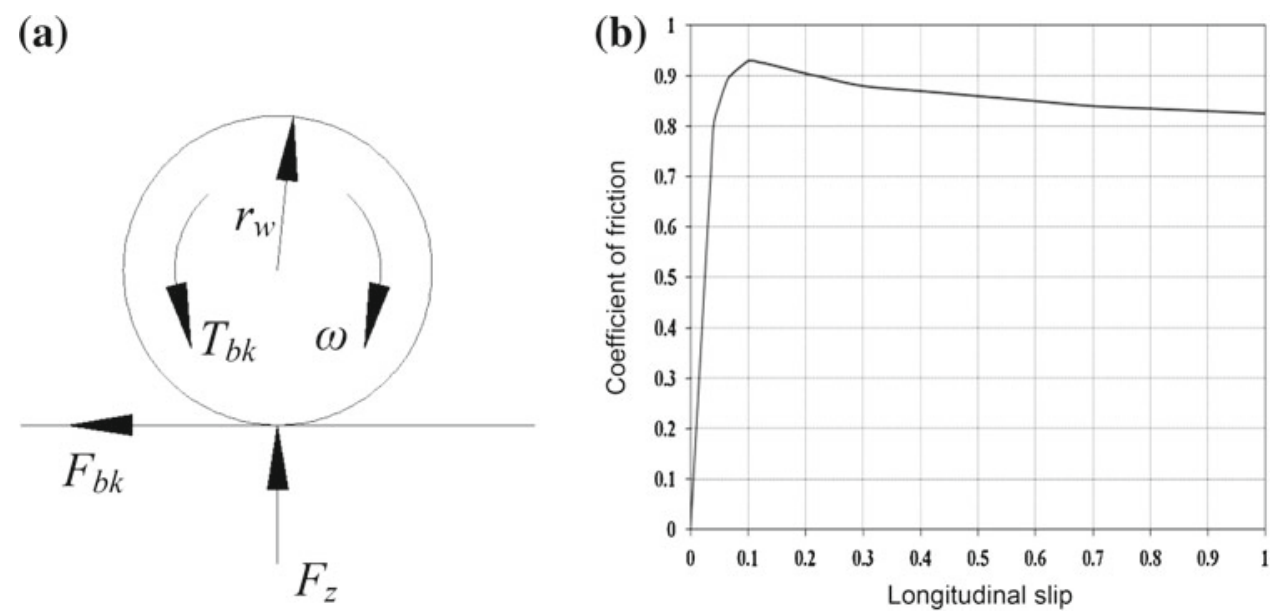

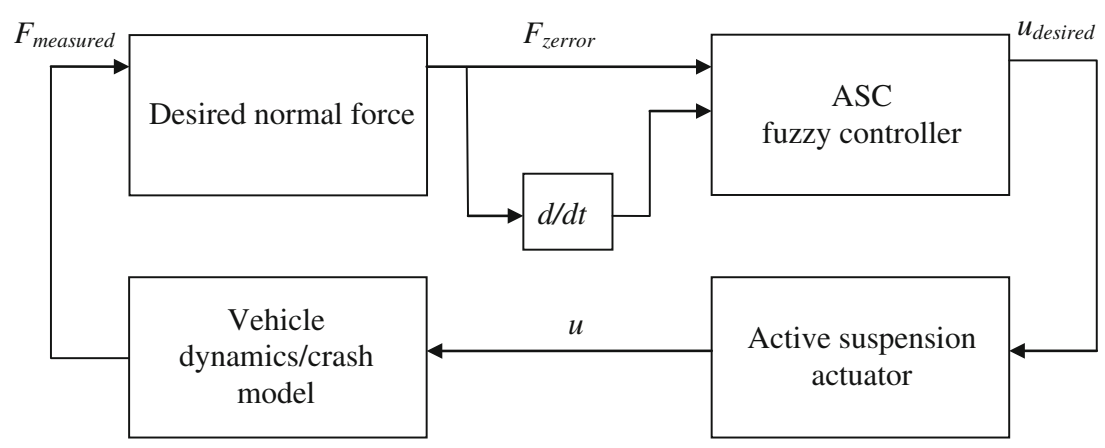

Eq. (43) and then the values of the slip ratio $\lambda$ can be estimated. The values of the slip ratio are then used to feed the ABS controller. This controller compares the error between the desired slip ratio (from 0.08 to 0.18 ) and the estimated one. Relative to the error comparison, the ABS controller turns the brake on/off which controls the braking torque $\mathrm{Tb}$ to sustain the coefficient of friction $\mu$ at its maximum values, thus the maximum braking force can be obtained.

The vertical forces $F_{z k}$ at each wheel can be written as follows:

$$
\begin{aligned}
& F_{z f R}=m g \cdot \frac{l_{r}}{l}+F_{S f R} \\
& F_{z f L}=m g \cdot \frac{l_{r}}{l}+F_{S f L} \\
& F_{z r R}=m g \cdot \frac{l_{f}}{l}+F_{S r R} \\
& F_{z r L}=m g \cdot \frac{l_{f}}{l}+F_{S r L}
\end{aligned}
$$

\subsection{Active suspension control system using a fuzzy logic controller}

The objective of the current controller is to increase the normal force $F_{z}$ on the front and rear wheels, before and during the crash, to increase the braking force $F_{b}$. In this case, the normal ASC, which is used for vehicle ride is deactivated. Figure 9 shows the schematic diagram of the ASC fuzzy logic controller.

The input variable (measured normal force $F_{z}$ ) is firstly compared with the target normal force $F_{z t}$ and the output is the error $\left(F_{\text {zerror }}\right)$. This error will then be integrated to get the change in error $\left(d F_{\text {zerror }} / d t\right)$. These two variables then will be feed to the fuzzy controller as input variables, $\left(F_{z e r r o r}\right)$ and $\left(d F_{\text {zerror }} / d t\right)$, which can be described as follows:

$F_{\text {zerror }}=F_{\text {measured }}-F_{z t}$

$\frac{d F_{\text {zerror }}}{d t} \approx \frac{\Delta F_{\text {zerror }}}{\Delta t}$

The output of the fuzzy controller is the desired actuation force which then be sent to the active suspension actuator. Related to the mechanical constraints of the active suspension system, the actual force element will be delivered to the vehicle model. The membership functions of the input and output variables are considered as a triangle shape as shown in Figs. 10 and 11, respectively.

The control rules for the proposed ASC fuzzy logic control are summarized in Table 2. Where NL denotes negative large, NS denotes negative small, Z denotes zero, PS denotes positive small, and PL denotes positive large. The defuzzification of output is carried out using the standard center 

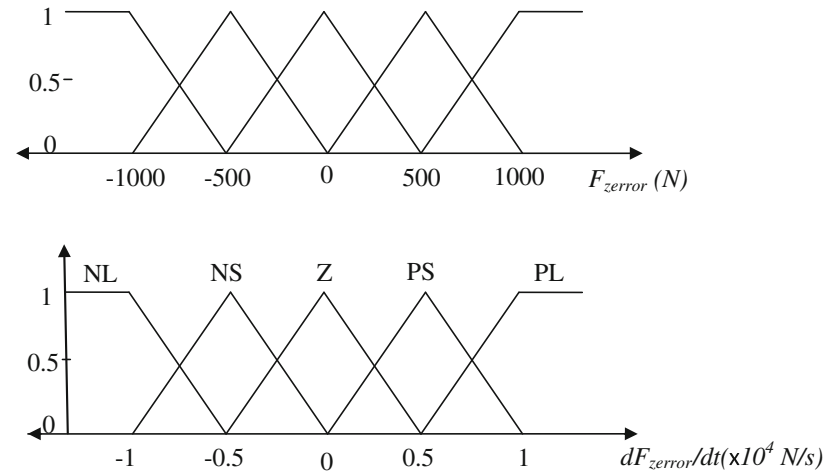

Fig. 10 Membership functions of the input variables (ASC)

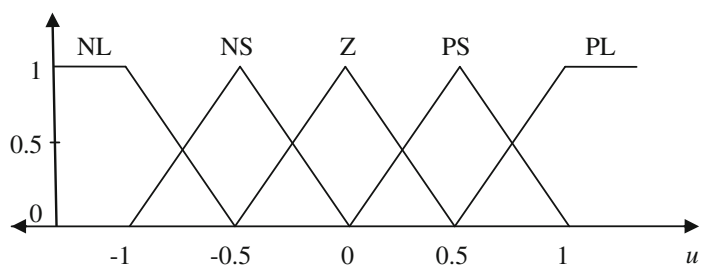

Fig. 11 Membership functions of the output variable (ASC)

of gravity (COG) defuzzification method. The maximum ASC force is considered to be $2000 \mathrm{~N}$ on each wheel with the maximum suspension travel limit of $\pm 100 \mathrm{~mm}$. The disadvantage of this controller is the high cost required to measure the normal force for each wheel.

Table 2 The fuzzy control rules of the ASC controller

\begin{tabular}{llllll}
\hline $\begin{array}{l}\text { The force element } \\
\text { of the ASC }(u)\end{array}$ & \multicolumn{5}{l}{ Change in error $\left(d F_{\text {zerror }} / d t\right)$} \\
\cline { 2 - 6 } & NL & NS & Z & PS & PL \\
\hline Error $\left(F_{\text {zerror }}\right)$ & & & & & \\
NL & PL & PL & PL & PS & Z \\
NS & PL & PL & PS & Z & NS \\
Z & PL & PS & Z & NS & NL \\
PS & PS & Z & NS & NL & NL \\
PL & Z & NS & NL & NL & NL \\
\hline
\end{tabular}

\subsection{Anti-pitch control system using a fuzzy logic controller}

The APC system is integrated with the ABS using the ASC to keep the vehicle in a horizontal position before the crash by applying an active force element on the front and rear wheels in upward and downward directions, respectively. A Mamdani fuzzy control scheme is used to control the vehicle in this case. Figure 12 shows a schematic diagram of the APC fuzzy logic controller.

The input variable actual pitch angle is firstly compared with the target pitch angle and the output is the error $\left(\theta_{\text {error }}\right)$. This error in the pitch angle will then be integrated to obtain the change in error $\left(d \theta_{\text {error }} / d t\right)$. The fuzzy controller inputs are the difference between the desired and the actual pitch angle $\left(\theta_{\text {error }}\right)$ and the change in error $\left(d \theta_{\text {error }} / d t\right)$ which can be described as follows:

$\theta_{\text {zerror }}=\theta_{\text {actual }}-\theta_{\text {target }}$

$\frac{d \theta_{\text {zerror }}}{d t} \approx \frac{\Delta \theta_{\text {zerror }}}{\Delta t}$

The output of the fuzzy controller is the desired actuation force which then be sent to the active suspension actuator. The membership functions of the input and output variables are considered as a triangle shape as shown in Figs. 13 and 14, respectively. The control rules for the proposed APC fuzzy logic control are summarized in Table 3 . The defuzzification of output is carried out using the standard COG defuzzification method.

\subsection{Under-pitch control system using a fuzzy logic controller}

The fifth case to be developed with the aid of the active suspension control (ASC) system is the UPC system. The idea of the UPC controller is to give the vehicle body negative pitch angle before the crash and try to maintain the vehicle in this case until it collides the barrier/other vehicle. The objective of the UPC system is to obtain the minimum pitching angle and acceleration of the vehicle body during the crash. The same controller used for the APC is also used for the UPC
Fig. 12 schematic diagram of the APC fuzzy logic controller

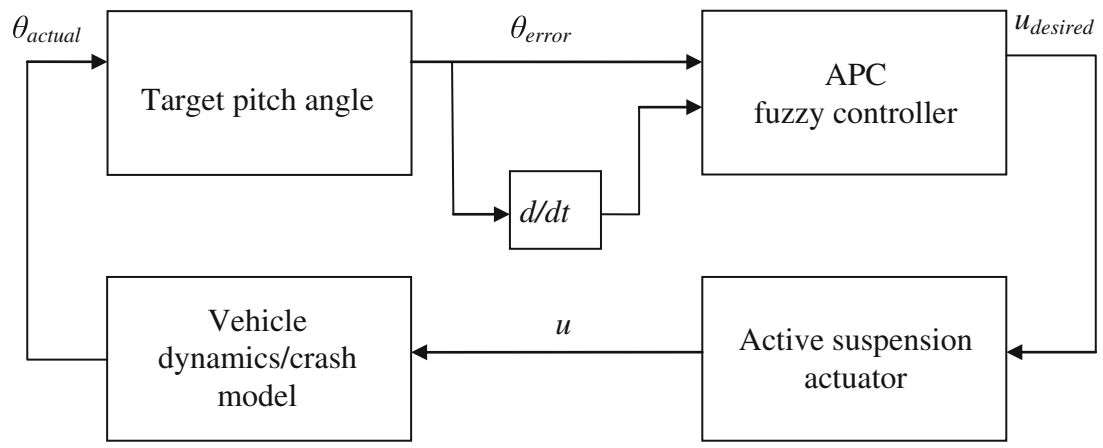



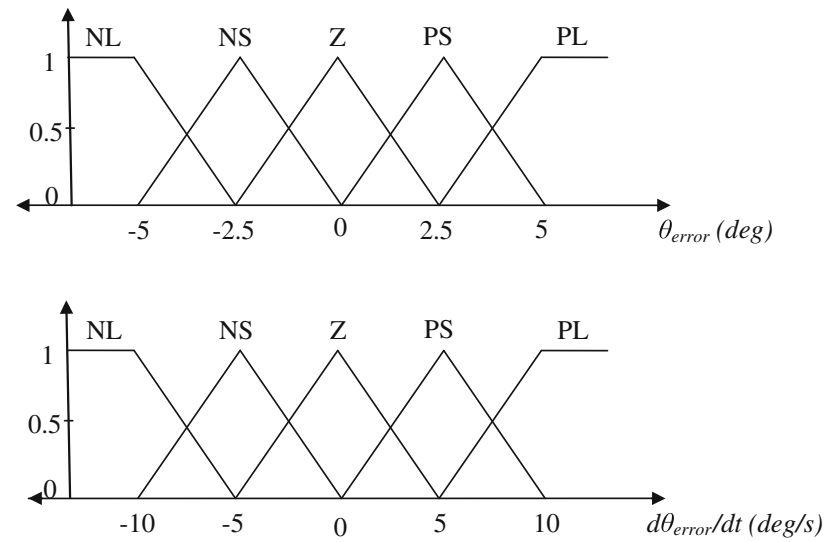

Fig. 13 Membership functions of the input variables

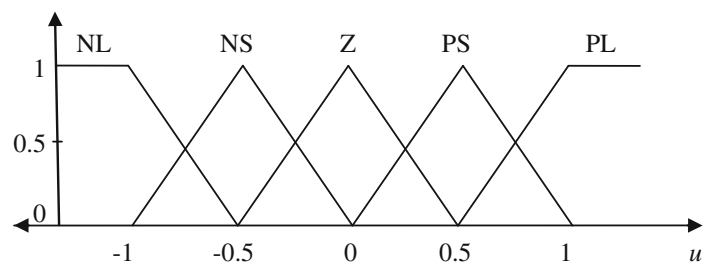

Fig. 14 Membership functions of the output variable

by changing the desired pitch angle from zero to desired negative value.

\section{Simulation results}

In this section, the model developed in the previous sections is used in the case of $50 \%$ offset crash scenario. The equations of motion have been solved using the central difference method with a constant time step of $0.001 \mathrm{~s}$ using the LabView software. While the ADAS detected that the crash is unavoidable at $1.5 \mathrm{~s}$ prior to the impact [11], the vehicle dynamics control systems (VDCS) will be applied in this short time prior the impact. The values of different parameters used in numerical simulations are given in Table 4

Table 3 The fuzzy control rules of the UPC controller

\begin{tabular}{llllll}
\hline $\begin{array}{l}\text { The force element } \\
\text { of the UPC }(u)\end{array}$ & \multicolumn{5}{l}{ Change in error $\left(d \theta_{\text {error }} / d t\right)$} \\
\cline { 2 - 6 } & NL & NS & Z & PS & PL \\
\hline Error $\left(\theta_{\text {error }}\right)$ & & & & & \\
NL & PL & PL & PL & PS & Z \\
NS & PL & PL & PS & Z & NS \\
Z & PL & PS & Z & NS & NL \\
PS & PS & Z & NS & NL & NL \\
PL & Z & NS & NL & NL & NL \\
\hline
\end{tabular}

Table 4 The values of the different parameters [3]

\begin{tabular}{ll}
\hline Parameter & Value \\
\hline$m$ & $1,200 \mathrm{~kg}$ \\
$m_{b}$ & $40 \mathrm{~kg}$ \\
$I_{y y}$ & $1,490 \mathrm{~kg} \mathrm{~m}^{2}$ \\
$I_{x x}$ & $350 \mathrm{~kg} \mathrm{~m}^{2}$ \\
$I_{z z}$ & $1,750 \mathrm{~kg} \mathrm{~m}^{2}$ \\
$I_{b z z}$ & $40 \mathrm{~kg} \mathrm{~m}{ }^{2}$ \\
$k_{S f R}=k_{S f L}$ & $18.25 \mathrm{kN} / \mathrm{m}$ \\
$k_{S r R}=k_{S r L}$ & $13.75 \mathrm{kN} / \mathrm{m}$ \\
$c_{f R}=c_{f L}$ & $1,100 \mathrm{~N} \mathrm{~s} / \mathrm{m}$ \\
$c_{r R}=c_{r L}$ & $900 \mathrm{~N} \mathrm{~s} / \mathrm{m}$ \\
$l_{f}$ & $1.185 \mathrm{~m}$ \\
$l_{r}$ & $1.58 \mathrm{~m}$ \\
$h$ & $0.452 \mathrm{~m}$ \\
$l_{a}$ & $1.2 \mathrm{~m}$ \\
$l_{b}$ & $0.85 \mathrm{~m}$ \\
$a=b$ & $0.8 \mathrm{~m}$ \\
$b_{i}=b_{o}$ & $0.8 \mathrm{~m}$ \\
\hline
\end{tabular}

[3]. The vehicle is adapted to collide with another vehicle at $55 \mathrm{~km} / \mathrm{h}$ speed for both vehicles. Prior collisions, the frontsprings forces are deactivated in the equations of motion. At the point of impact, the vehicle collides the other vehicle by the same speed. The front-end "springs" forces are re-deactivated at the end of collision (vehicle velocity equal zero/negative values) and the behavior of the vehicle in postcollision is captured. Front-end deformation, vehicle body acceleration, pitching and yawing are depicted to assess the crash behavior of each vehicle.

\subsection{Response of the control systems}

The response of the controller output of the ASC, APC, and UPC systems are presented in this section. At a collision speed of $55 \mathrm{~km} / \mathrm{h}$ in a vehicle-to-vehicle collision scenario, the output responses of the ASC system before and after the crash are shown in Fig. 15. The output of the active suspension force element on the front and rear wheels is fluctuating to track the desired normal force as shown in Fig. 15 and 16. The desired normal force is selected, related to the physical parts, to be as maximum as possible. After about $0.17 \mathrm{~s}$ the active force on the rear wheels reaches the minimum limit value of $-2000 \mathrm{~N}$ and it is not increasing again before or after the crash. The active force on the front wheels decreases at the point of impact and then increases to reach the maximum limit value of the active suspension system as of $+2000 \mathrm{~N}$ and then kept constant till the end of the collision.

The corresponding normal force $\left(F_{z}\right)$ on the front and rear wheels with and without (only ABS is applied) the ASC is depicted in Fig. 16. While the normal force on the front and 
Fig. 15 The output response of the fuzzy controller (ASC)

Fig. 16 Normal force on the front and rear wheels (with and without ASC)

Fig. 17 Vehicle body pitch angle before and after crash
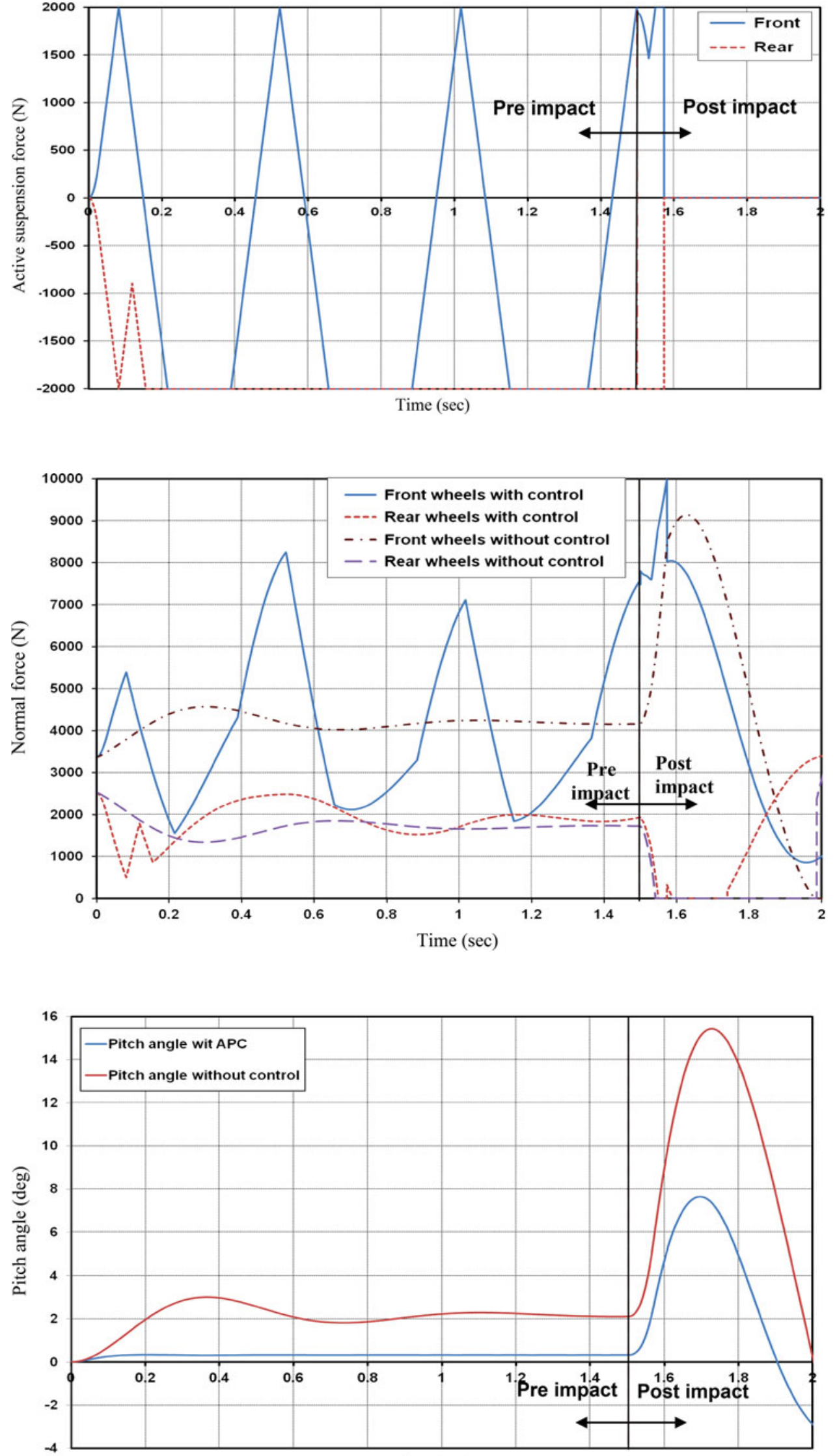

Time (sec) 
Fig. 18 The output response of the fuzzy controller of the APC system

Fig. 19 The output response of the fuzzy controller of the UPC system
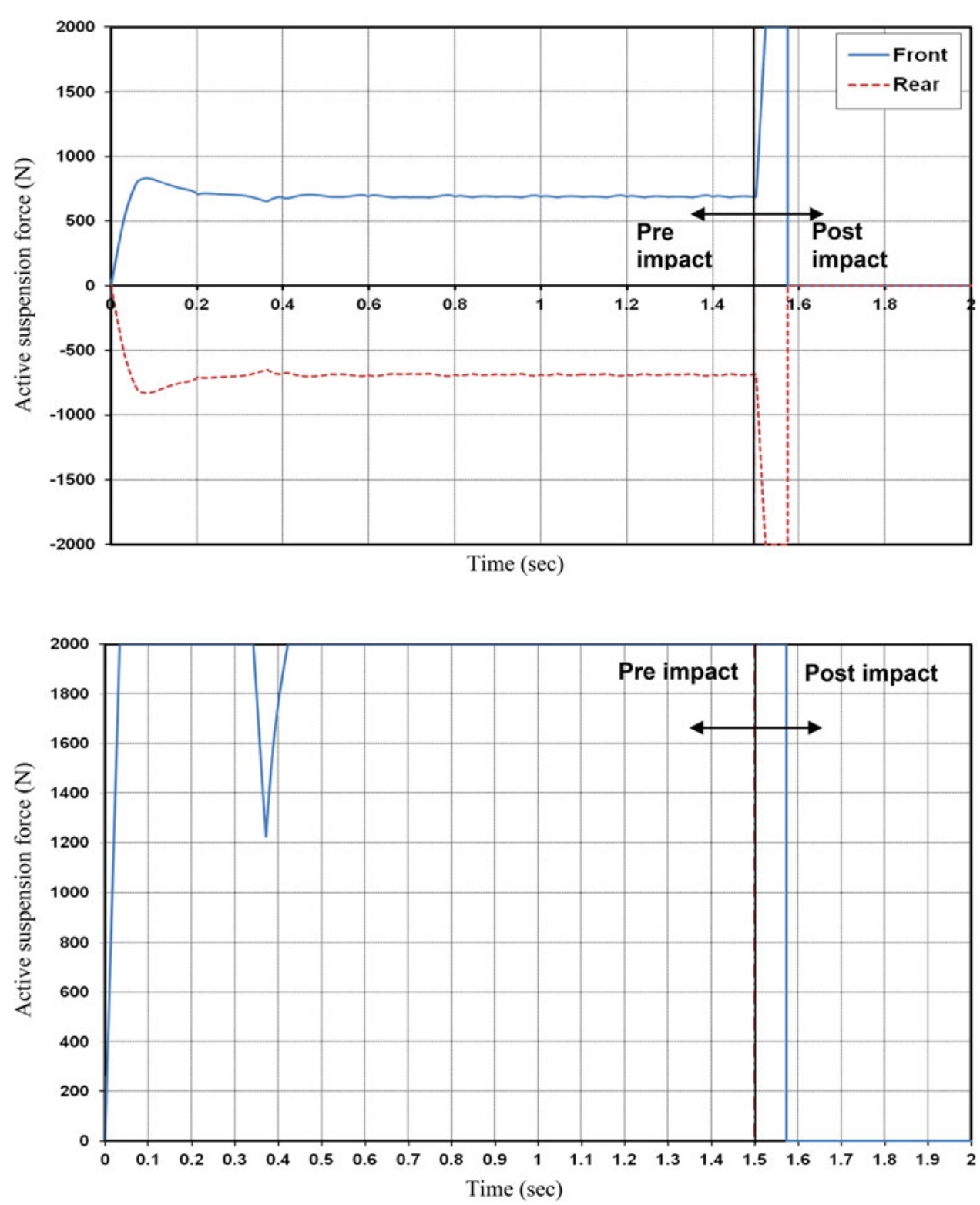

rear wheels with ASC is fluctuating to be smaller or greater than the normal force without ASC, the mean force values in case of ASC are greater than the normal force without ASC. The mean force value here is measured during $1.5 \mathrm{~s}$ before collision. One of disadvantage of the fuzzy controller is its instability response, and the fluctuation to the normal force is the result from this instability. However, using this controller leads to a noticeable increase of the braking force and the stopping distance before the crash [3,12]. This also causes a reduction of the deformation of the front-end structure after the crash. Just after the point of impact, the normal force on the rear wheels in the above two cases is reduced rapidly to reach a value of zero (when the rear wheels left the ground), while the normal force on the front wheels is increased very quickly. It is worth noting that the period in which the rear wheels leave the ground is shorter when the ASC is applied than in the case of ABS applied alone. It is observed that when the ASC is used, the maximum normal force on the front wheels occurs at the end of the collision (about $0.08 \mathrm{~s}$ after collision); at this point the active control is deactivated because the vehicle speed is equal to zero. On the other hand, in the case of ABS is applied alone, the maximum normal force on the front wheels occurs after about $0.05 \mathrm{~s}$ by the end of the crash as shown in the Fig. 16.

The vehicle pitch angle and the output response of the APC controller are shown in Figs. 17 and 18, respectively. First, the vehicle body starts to take a positive pitch angle due to the braking force (1.5 s before the crash). Therefore, the input 
Fig. 20 Vehicle body pitch angle before and after crash of the UPC system
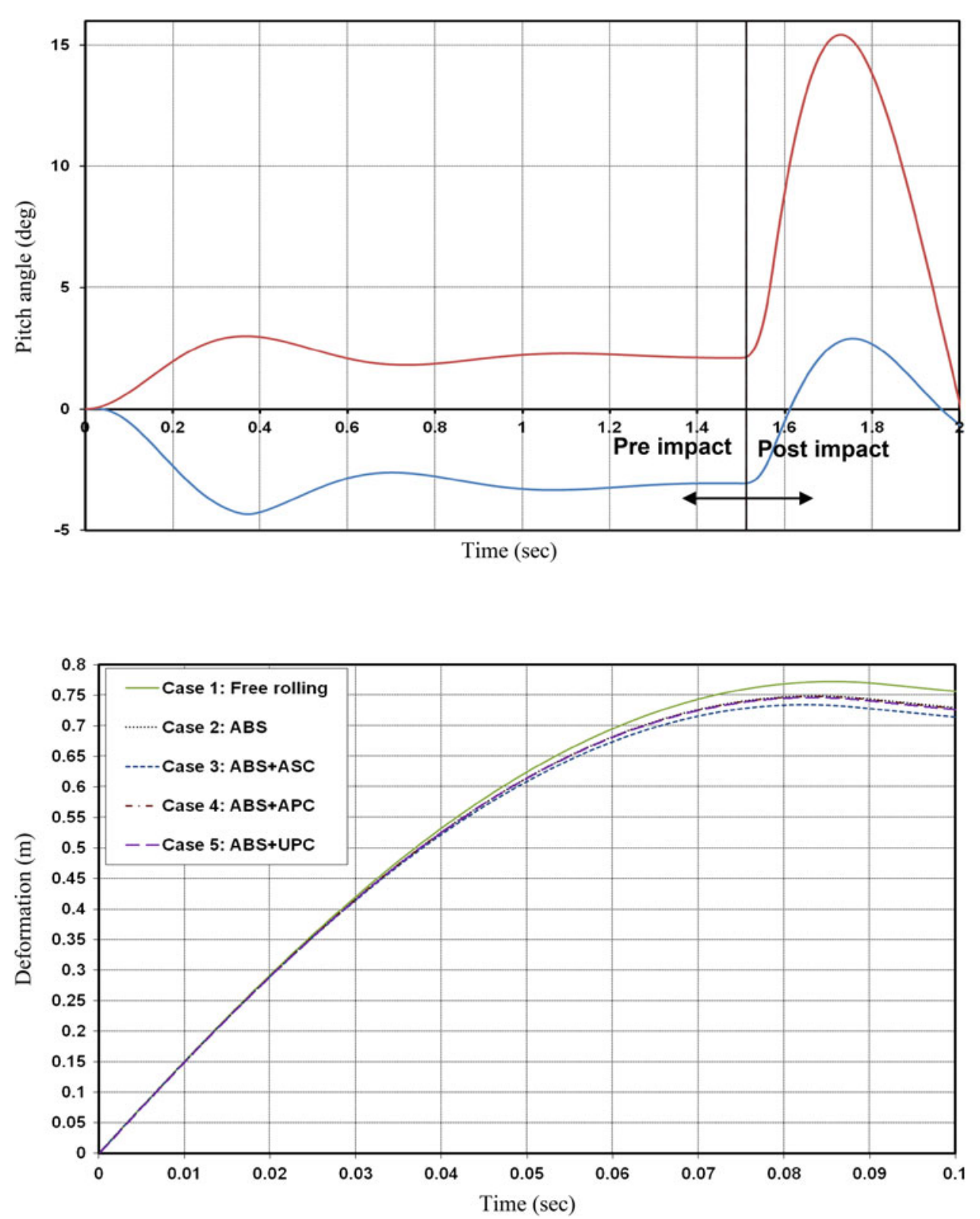

Fig. 21 Deformation of the front-end structure, vehicle (a) error (the difference between the desired and actual pitching angle) and the change in the input error are increased. The APC controller then activates the suspension actuator to apply an active vertical force in the upward direction on the front wheels and in the downward direction on the rear wheels. Following this, once the vehicle pitching rate reaches zero, the maximum pitch angel is very close to the desired value (zero in this case), the output force is almost remained constant at this value as shown in Fig. 18. When the vehicle reaches the barrier, the pitching angle increased very quickly due to the crash, and the controller output reaches its maximum value of $+2000 \mathrm{~N}$ for front wheels and $-2000 \mathrm{~N}$ for rear wheels.
The output actuation force of the UPC system on the vehicle's front wheels is depicted in Fig. 19. At the beginning when the controller is applied (1.5 s prior the collision), the error (the difference between the desired and actual pitching angle) is large positive compared with the desired negative value. Therefore, the output force of the system increases rapidly when the controller is activated to reach its maximum allowable active force. At time of $3.5 \mathrm{~s}$, the active force drops for a short time (when the pitch angle is close to the desired value of negative $5^{\circ}$ ); following this, the active force remains constant at the maximum value to the end of the collision. The response of the output actuation force on the rear wheels is exactly the reflection of the force/time curve of 
Fig. 22 Deformation of the front-end structure, vehicle (b)

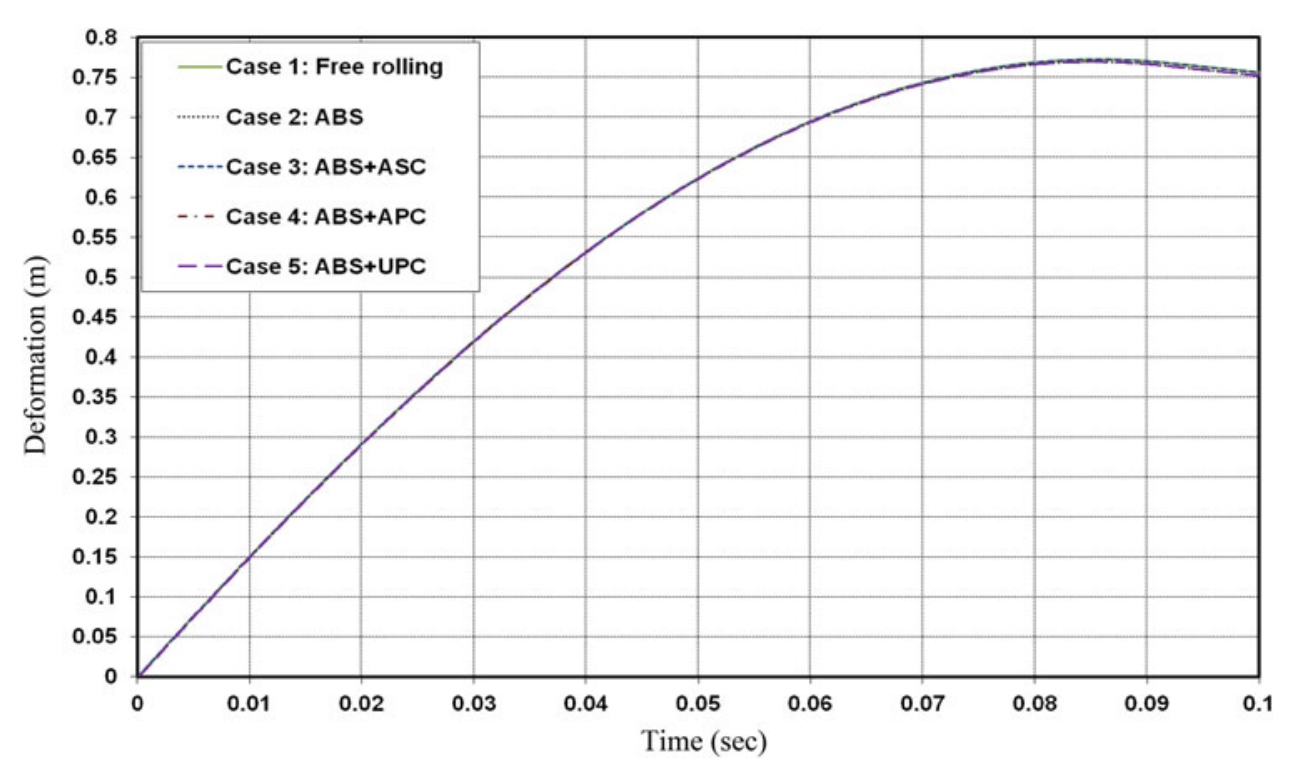

Fig. 23 Vehicle body deceleration, vehicle (a)

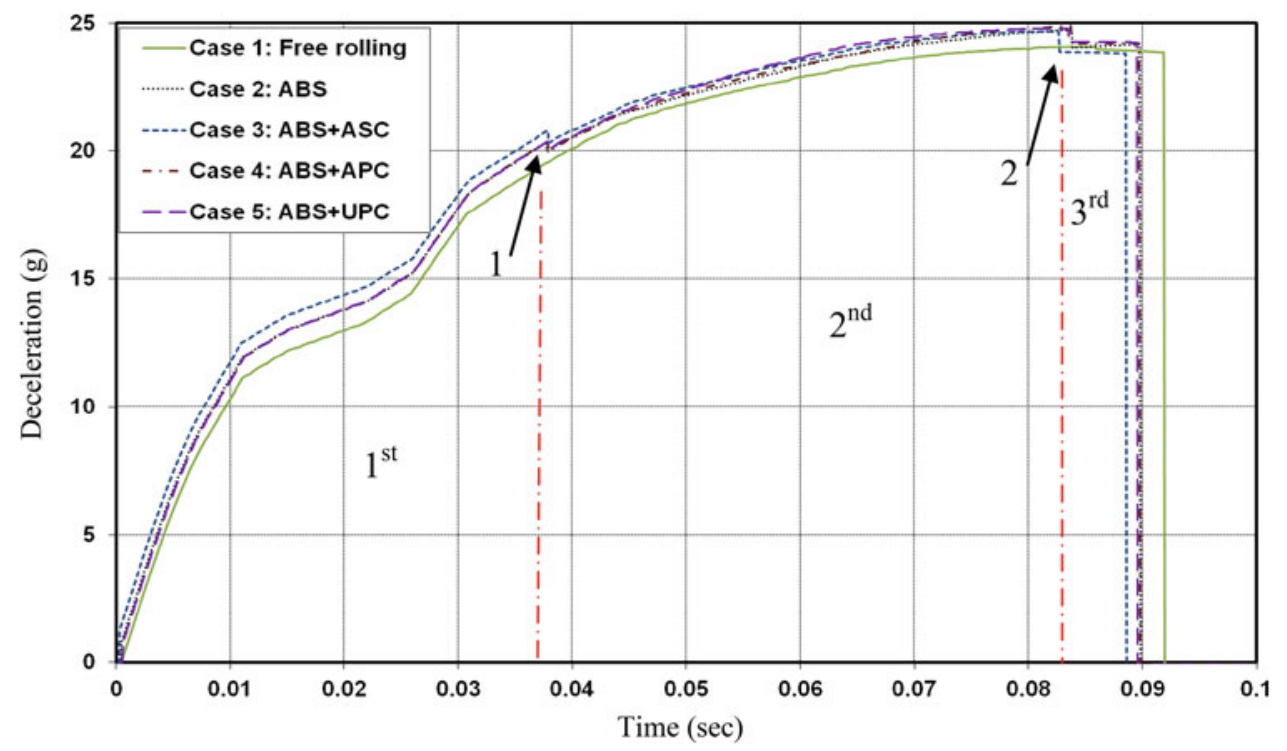

the front wheels, shown in Fig. 19, about x-axis. The pitching angle of the vehicle body before the crash is shown in Fig. 20. The vehicle body takes a rapid negative pitch angle at the beginning of the controller activation to be close to the desired pitch angle of negative $5^{\circ}$. When the vehicle pitch angle is close to this negative peak value, it returned due to the opposite force generated by suspension springs. The vehicle then remains steady with a small bouncing at around negative $3^{\circ}$ until the point of impact and then increased very rapidly due to the collision.

\subsection{Vehicle-to-vehicle crash results}

The effect of the different cases of VDCS on vehicle collision mitigation is investigated. In addition, the effect of the control systems on the other vehicle (vehicle b) is discussed. In the following figures the crash event is considered to be started at time $\mathrm{t}=$ zero. Figure 21 shows the impacted side of the front-end structure's deformation-time histories for vehicle a for all different VDCS cases. It is noticed that the deformation increased to reach its maximum value (different for each case) and then decreased slightly due to front-end springs rebound. The minimum deformation is obtained in the case 3 when the ASC is applied along with ABS. The maximum reduction of $50 \mathrm{~mm}$ is observed in this case and a reduction of $30 \mathrm{~mm}$ is shown in case 5 , while a reduction of about $25 \mathrm{~mm}$ is obtained in cases 2 and 4 compared with the free rolling case. The integrated control of the ASC with the ABS aims to increase the braking force by increasing the vertical load to obtain a minimum stopping distance. It 
Fig. 24 Vehicle body deceleration, vehicle (b)

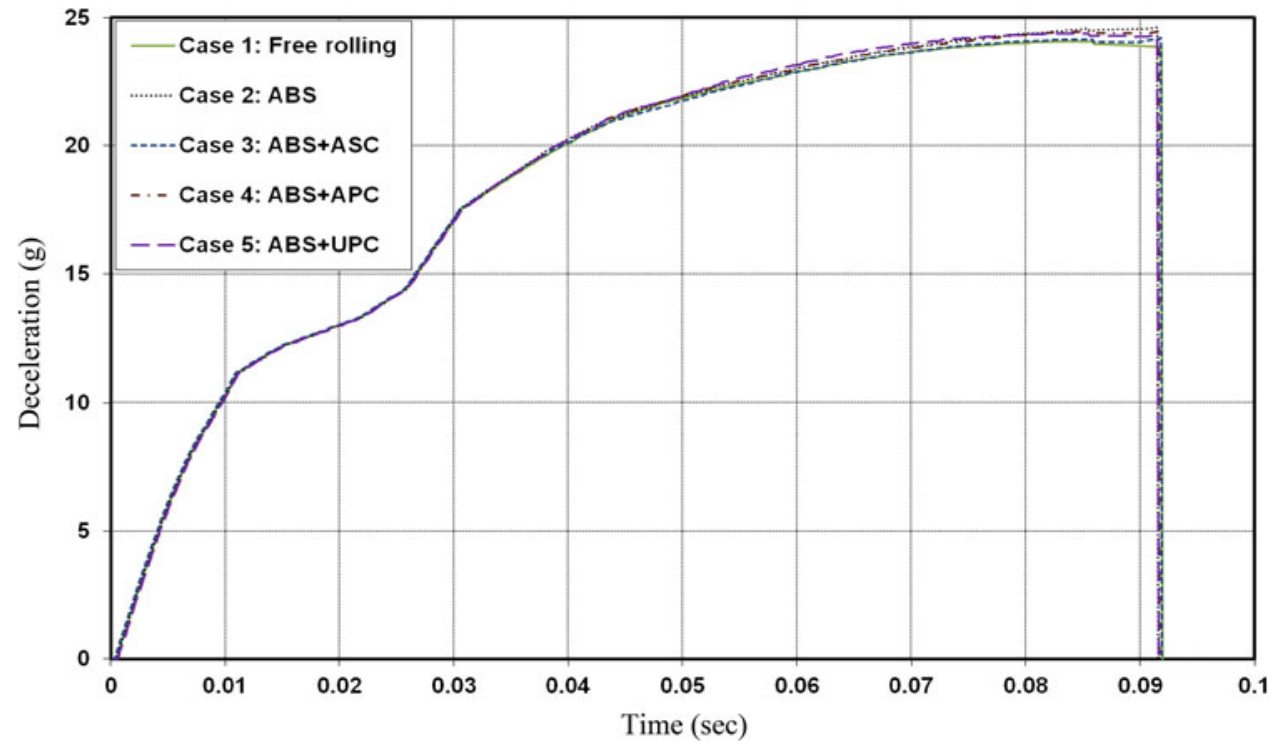

Fig. 25 Vehicle body pitch angle, vehicle (a)

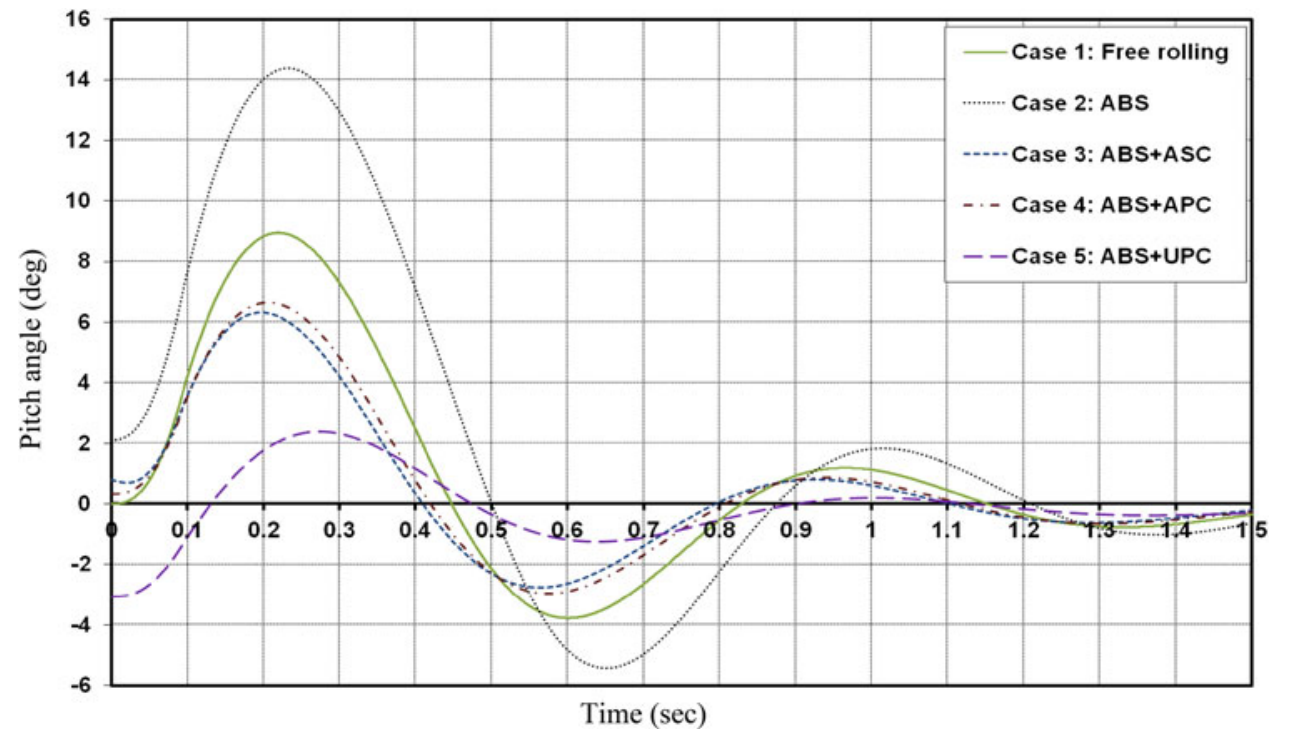

is worth mentioning that the application of the ASC control system (case 3 ) helps reducing the maximum deformation of the front-end structure as shown in Fig. 21.

Figure 22 shows the front-end structure's deformationtime histories for vehicle $\mathbf{b}$ in all different VDCS cases. Because the two vehicles are identical and both collide at the same speed, it is clearly shown that the simulation results of the free rolling case are almost the same. The maximum deformation is almost the same with very small and insignificant values for all cases of VDCS, and this means the control systems have no great effect on the front-end deformation of the other vehicle during the collision.

The deceleration-time histories of the vehicle body for all cases for vehicle a are presented in Fig. 23. The decelerationtime history can be divided to three stages. The first stage represents the increase of the vehicle's deceleration before the front left wheel reach the barrier. In this stage the highest deceleration value is observed in case 3. In the other cases, a slight higher deceleration is also noticed compared with the free rolling case. In the second stage, the front left wheel reaches the barrier and stop moving, therefore its braking effects is vanished. At the beginning of this stage a rapid reduction in the vehicle body deceleration occurs (arrow 1, Fig. 23); this deceleration drop does not appear in the free rolling case while there is no applied braking. During the second stage, it is noticed that the minimum deceleration is still in case 1 , while the maximum deceleration is almost the same for all other cases. At the end of this stage, the vehicle stops and starts moving in the opposite direction. In addition, the braking force changes its direction and 
Fig. 26 Vehicle body pitch acceleration, vehicle (a)
Fig. 27 Vehicle body pitch angle, vehicle (b)
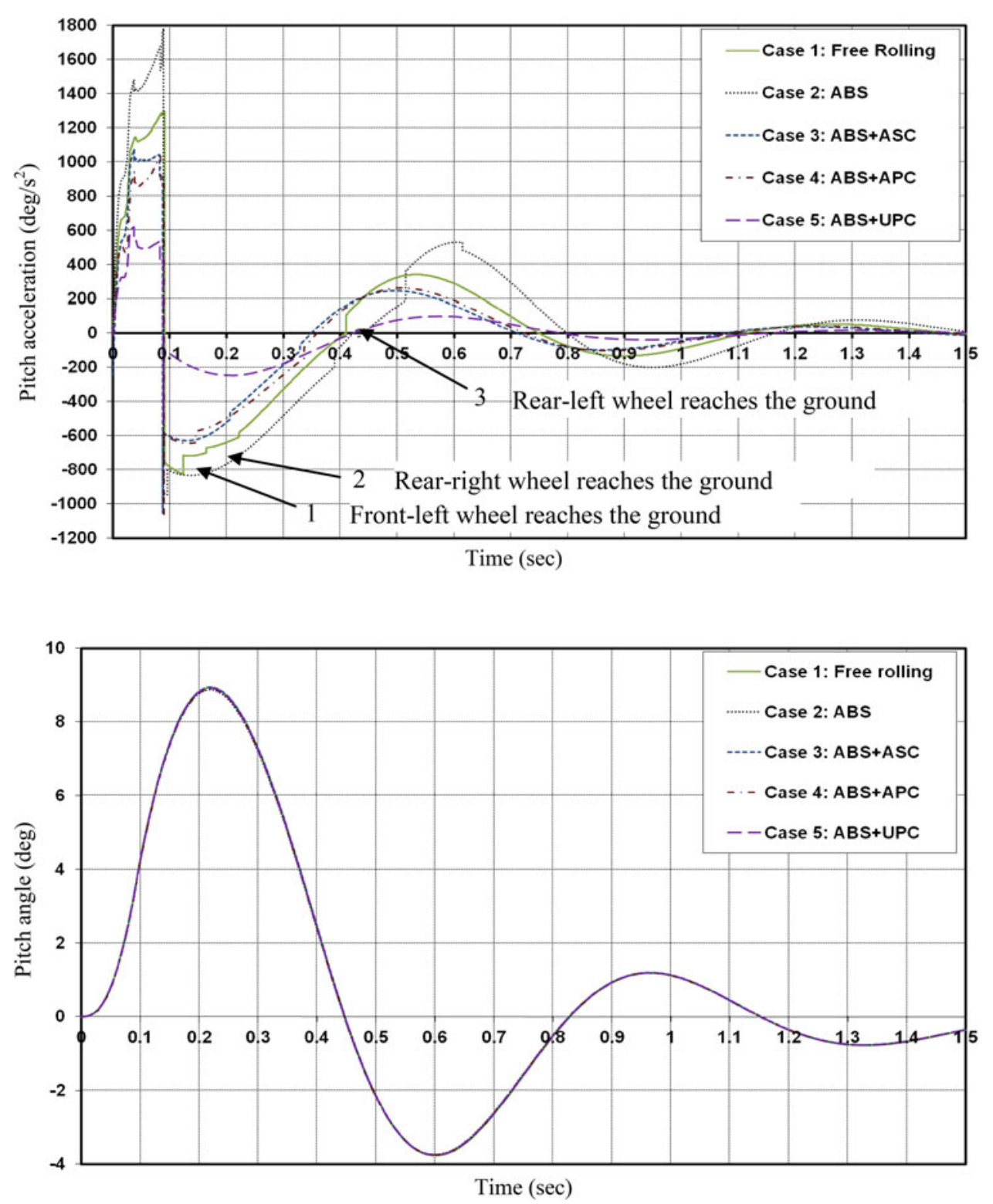

another drop in the vehicle deceleration is noticed as shown in Fig. 23, (arrow 2). At the third stage, a condition of allowing the front-end springs to be rebounded for a very short time is applied during the simulation analysis. During this stage, the vehicle moves back and the deformation of the front-end decreases. At the end of this stage, the non-linear front-end springs are deactivated and the vehicle's deceleration is suddenly dropped to a value of zero. This fast drop is due to the assumption of immediate stopping the effect front-end springs after very short time of rebound.

An insignificant increase of the vehicle deceleration for all VDCS cases is observed in the other vehicle $\mathbf{b}$ compared with the free rolling case as shown in Fig. 24. The maximum values of the vehicle deceleration in vehicle $\mathbf{b}$ are also almost the same for all the VDCS cases.
Figure 25 shows the vehicle's pitch angle-time histories for all cases for vehicle a. The VDCS is applied $1.5 \mathrm{~s}$ before collision, therefore, the vehicle body impacts the barrier at different values of pitch angles according to each case as shown in Fig. 25. The vehicle's pitch angle then reaches its maximum values (normally after the end of crash) according to each case. Following this, the pitch angle reduced to reach negative values and then bounces to reach its steadystate condition. In the offset crash scenario, vehicle body pitching angle is generated due to the difference in impact forces between the upper and lower front-end members of the impacted side in the free rolling case. Additional pitching moment is generated from the braking force in the other VDCS cases. The maximum pitch angle is observed in case 2 followed by case $1,4,3$ and finally case 5 . In case 5, a 
Fig. 28 Vehicle body pitch acceleration, vehicle (b)
Fig. 29 Vehicle body yaw velocity, vehicle (a)
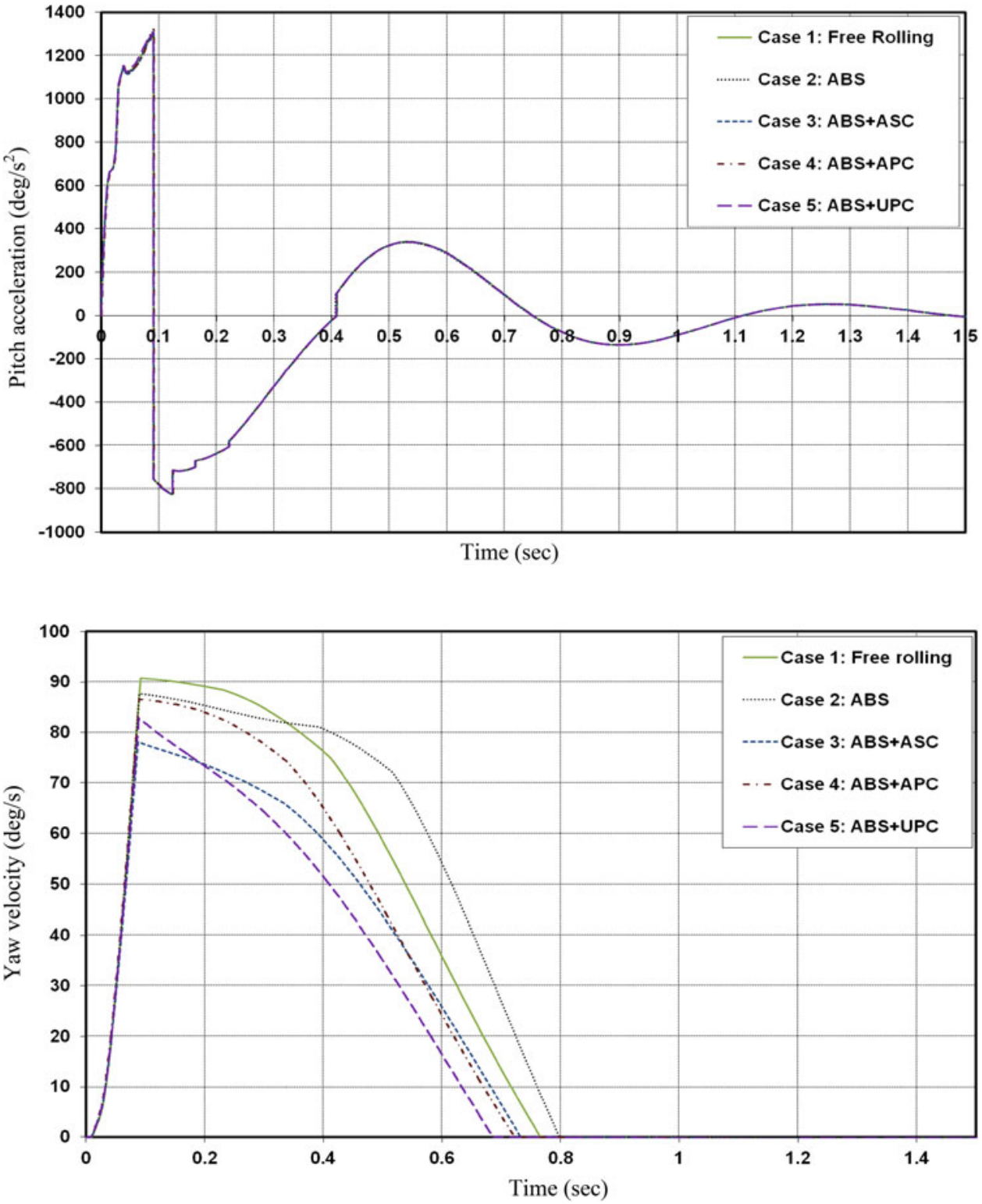

notable reduction of about $6.5^{\circ}$ compared with case 1 and about $12^{\circ}$, compared with case 2 are observed.

The vehicle body pitching acceleration is also depicted in Fig. 26 for all cases for vehicle a. A rolling moment of the vehicle body is generated during the crash due to the different values of the component of the left frontal springs' forces in $y$ direction and from the friction between the ground and the tires due to the yaw motion. At the end of the collision, the pitching and rolling moments are ended and the vehicle is controlled by the tires and suspension forces. The vehicle's rear wheels left the ground during the vehicle pitching and the left wheels (front and rear) left the ground as well during the vehicle rolling. At this moment, three wheels of the vehicle are not contacted with the ground with different distances.
This explains the different sudden changes of the vehicle pitching acceleration when each wheel re-contact the ground (look at the arrows referred to case 1 in Fig. 26).

The vehicle maximum pitching acceleration is observed in cases 2 whilst the lowest value is detected in case 5 (ABS + UPC). Compared with case 1 (free rolling) and case 2 (ABS), a reduction of about 670 and about $950^{\circ} / \mathrm{s}^{2}$, respectively, are obtained in case 5 (ABS + UPC).

Similarly, the pitch angle and pitch acceleration-time histories for vehicle b are depicted in Figs. 27 and 28, respectively. It is noticed that there is no difference between the results of the five crash scenarios. That means the different applied cases of the VDCS on vehicle a do not affect the pitching event of vehicle $\mathbf{b}$ in case of offset collision. 
Fig. 30 Vehicle body yaw acceleration, vehicle (a)
Fig. 31 Vehicle body yaw angle, vehicle (a)
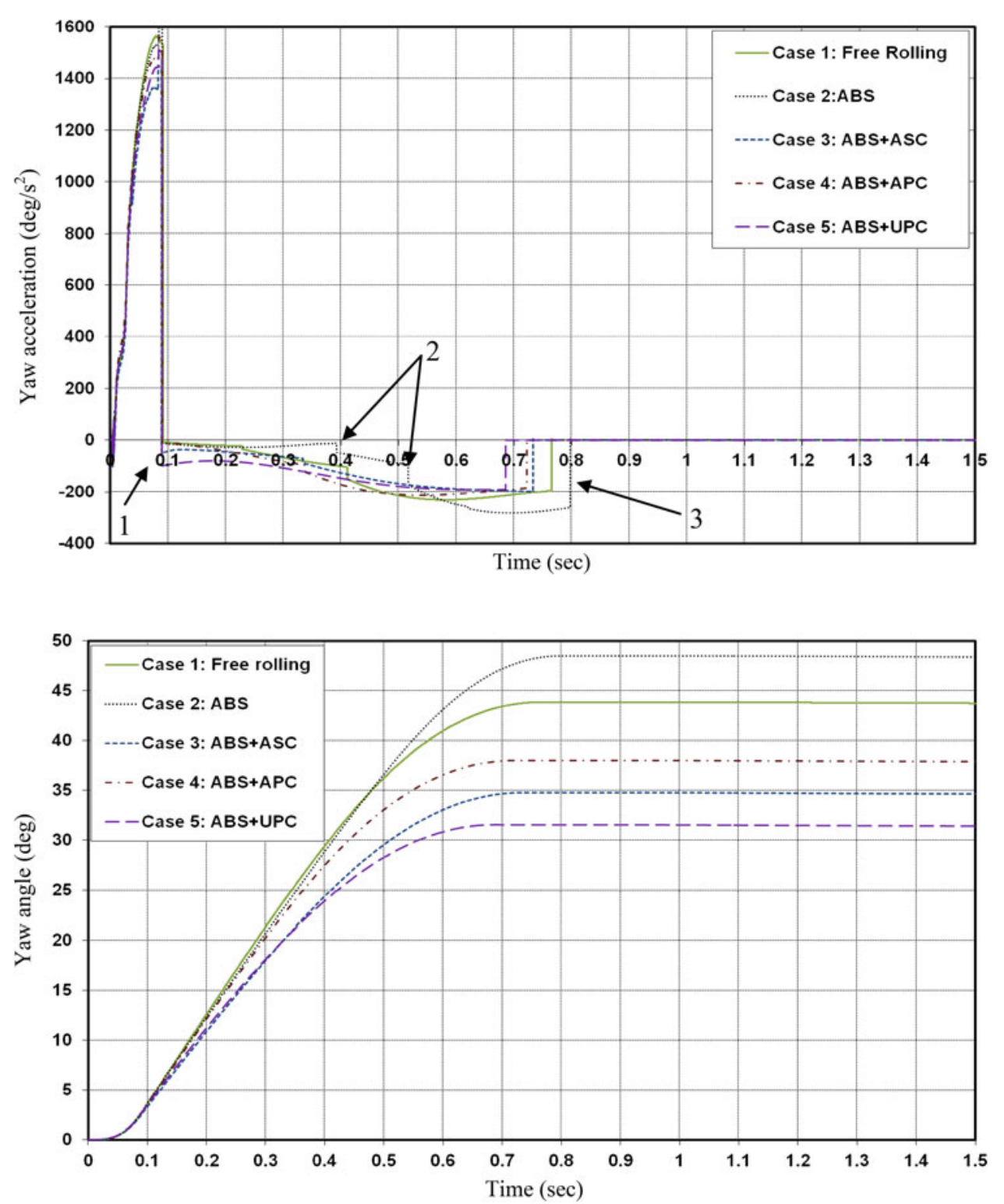

Figure 29 shows the vehicle yaw velocity-time histories for all five cases for vehicle a. The vehicle yaw velocity is equal to zero before the crash then it changes in three different stages: firstly, it increases rapidly to reach its maximum value; secondly it decreases slowly for a different period of time related to each case; and thirdly it decreases gradually to reach zero. In the first stage, the rapid increase in the yaw velocity is due to the high yawing acceleration (see Fig. 30) caused by the one side impacted member. At the end of collision, the rear wheels left the ground due to the vehicle pitching and the front-left wheel left the ground due to the vehicle rolling and hence the vehicle is controlled by the front-right wheel only. In the second stage, the decrease in the vehicle's yaw velocity occurred due to the friction force between the front-rear tire and the ground. The period of this stage is different for each case and it mainly depends on the maximum pitching angle. During the second stage, the front-left wheel re-contacts the ground. Stage 3 begins when the rear wheels starts contacting the ground generating yaw moments in the opposite direction. This is causing a reduction of the vehicle yawing velocity with a higher rate than the decreasing of velocity rate in the second stage. A reduction of the maximum yawing velocity $\left(10^{\circ} / \mathrm{s}\right)$ is observed in cases 3 and 5 , while a reduction of about $5 \% \mathrm{~s}^{2}$ is obtained for the other cases of VDCS.

Vehicle body yaw acceleration-time histories are depicted in Fig. 30. The maximum yaw acceleration is observed in case 1 (free rolling) and the minimum yaw acceleration is also observed in cases 3 and 5. At the end of colli- 
Fig. 32 Vehicle body yaw velocity, vehicle (b)

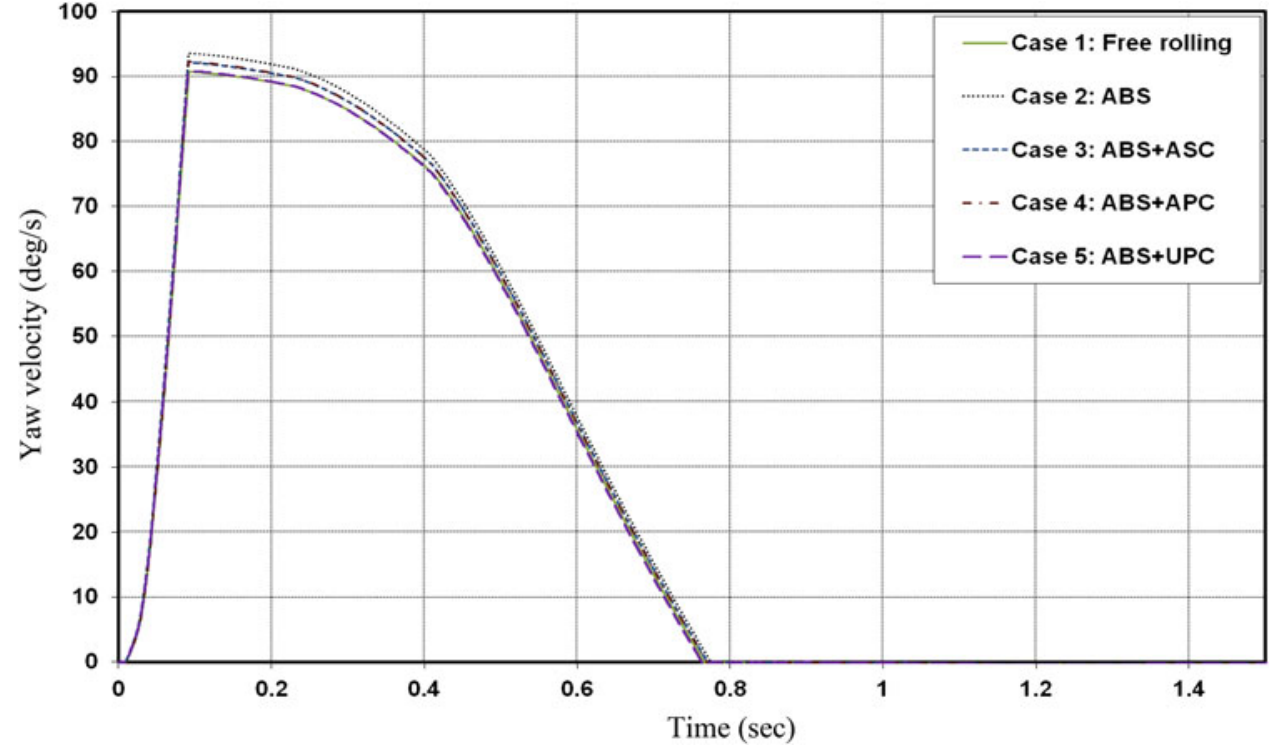

Fig. 33 Vehicle body yaw acceleration, vehicle (b)

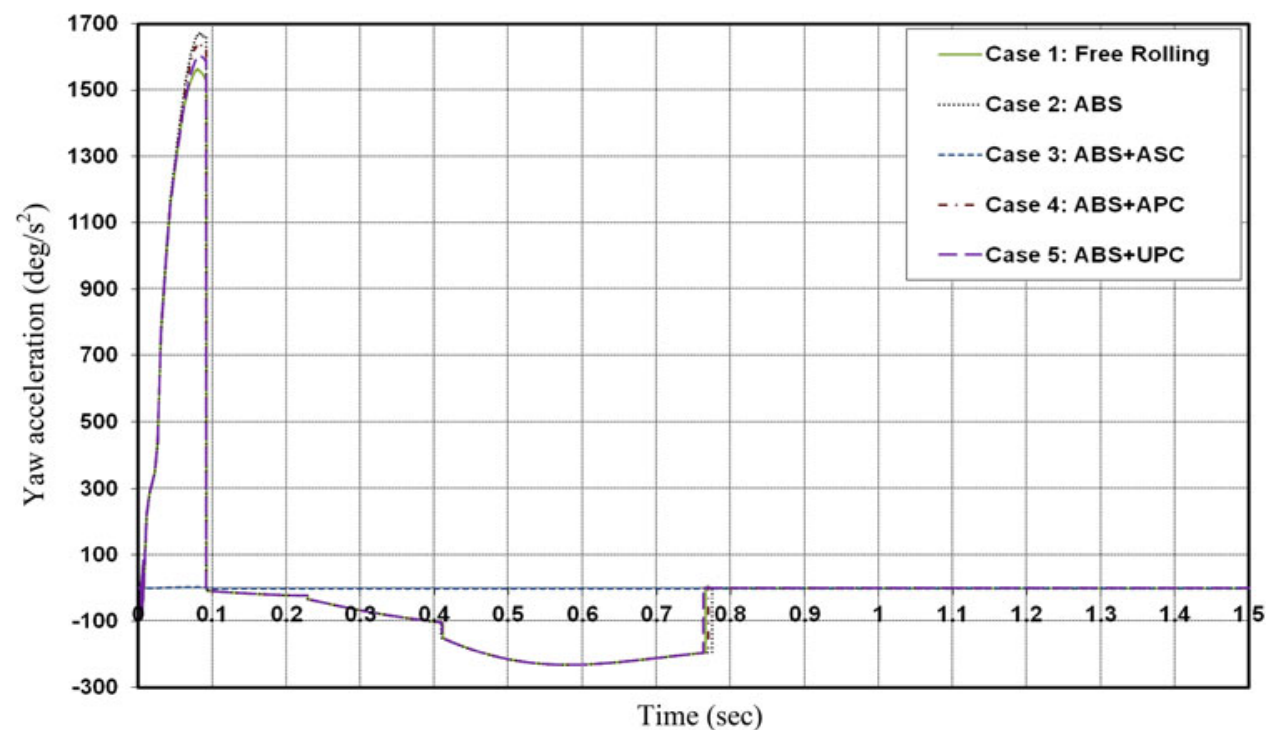

sion, the vehicle is controlled by the front-left wheel only, as mentioned before, trying to hinder the yawing motion. Accordingly, a negative yawing acceleration is generated with different small values related to each case as shown in Fig. 30 (arrow 1). These negative values of the vehicle yaw acceleration increase slowly with time producing two sudden drops of acceleration (arrow 2) once the right-rear wheel and the left-rear wheel re-contact the ground, respectively. These drops are not shown in case 5 because the rear wheels do not leave the ground in this case. When the vehicle yawing ends and the yaw speed reaches zero, the yaw acceleration returns to zero as well as shown in Fig. 30 (arrow 3).

Figure 31 shows the vehicle body yaw angle-time histories for all cases for vehicle a. It is found that the maximum yaw angle of $49.3^{\circ}$ is noticed in case 2 (ABS) while the minimum yaw angle of $36.8^{\circ}$ is noticed in case 5 (ABS + UP). The maximum value of the vehicle yaw angle depends on the maximum yaw acceleration and the vehicle pitch angle for each case. It is worth mentioning that reducing the maximum vehicle body yaw angle reduces the risk of the car side-impact by any obstacles on the road.

The yawing event of the vehicle $\mathbf{b}$, which is not equipped by the VDCS, is affected by vehicle a once different control systems are applied. The maximum yaw velocity of vehicle b is increased for all cases compared with the free rolling case, except in case 5, as shown in Fig. 32.

Figure 33 shows the yaw acceleration of the vehicle $\mathbf{b}$. It is observed that the maximum yaw acceleration is also increased for all cases compared with the free rolling case 
Fig. 34 Vehicle body yaw angle, vehicle (a)

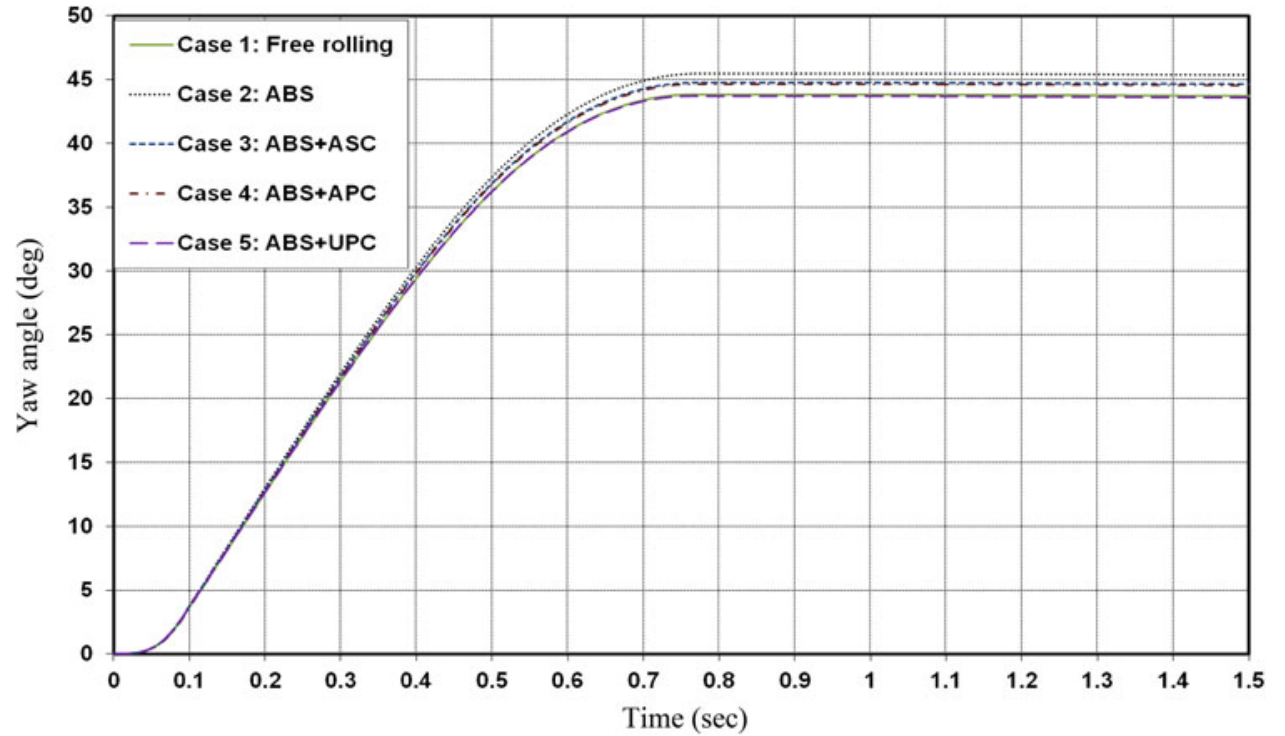

by different values related to each case. In the same manner, the maximum yaw angle of the vehicle $\mathbf{b}$ is increased for all cases by different values (from 1.5 to $2^{\circ}$ ) related to each case, except in case 5 as shown in Fig. 34.

\section{Summary and conclusions}

In this research, the influences of vehicle dynamic control systems on the vehicle collision are comprehensively investigated since VDCS plays important roles in vehicle safety, ride and stability. A unique 3-D vehicle dynam$\mathrm{ics} / \mathrm{crash}$ mathematical model is developed. This model combines vehicle crash structures and vehicle dynamics control systems. The results obtained from different applied cases show that the VDCS affect the crash situation, by different ratios related to each case, positively. The deformation of the vehicle front-end structure is reduced when the ASC is applied, and hence helps protecting the occupant inside the vehicle compartment. The vehicle body deceleration is insignificantly changed within the applied cases. The vehicle pitch angle and its acceleration and yaw angle and its acceleration are dramatically reduced when the UPC system is applied. While the reduction on the vehicle pitching helps reducing the head injury, the reduction in the maximum yaw angle helps reducing the risk of hitting another object on the road due to the vehicle rotation.

Acknowledgments The authors would like to thank the Egyptian Government and the Faculty of Engineering, Ain Shams University for supporting this research. The authors also acknowledge with sadness, the contribution of Prof. Dave Crolla who has passed away during the period of this research.

\section{References}

1. Mao Y, Zhang Y, Liu T (2010) The sliding mode controller for automotive ABS based on the fuzzy neural network. In: Sixth international conference on natural computation (ICNC), USA, 10-12 August 2010, pp 1410-1413

2. Sánchez-Torres JD, Loukianov, AG, Ruiz-León J, Rivera J (2011) ABS+ active suspension control via sliding mode and linear geometric methods for disturbance attenuation. In: 50th IEEE conference on decision and control and European control conference (CDC-ECC), 12-15 December 2011

3. Alleyne A (1997) Improved vehicle performance using combined suspension and braking forces. Veh Syst Dyn 27(4):235-265

4. Ting C, Tzuu-Hseng S, Kung L (1995) Design of fuzzy controller for active suspension system. J Mechatron 5(4):365-383

5. Tamura M, Inoue H, Watanabe T, Maruko N (2001) Research on a brake assist system with a preview function. In: SAE international, Warrendale, PA. SAE Technical Paper 2001-01-0357

6. Alirezaei M, Corno M, Ghaffari A, Kazemi R (2012) A new approach to the design of coordinated road departure avoidance systems. J Multi-body Dyn 226(1):45-60

7. Chang JM, Ali M, Craig R, Tyan T, El-bkaily M, Cheng J (2006) Important modeling practices in CAE simulation for vehicle pitch and drop. In: SAE international, Warrendale, PA. SAE Technical Paper 2006-01-0124

8. Hogan I, Manning W (2007) The use of vehicle dynamic control systems for automotive collision mitigation. In: Third Institution of Engineering and Technology conference on automotive electronics, Warwick, 28-29 June 2007, pp 1-10

9. Ting W, Lin JS (2004) Nonlinear control design of anti-lock braking systems combined with active suspensions. In: Fifth Asian control conference, vol 1, Melbourne, 20-23 July 2004, pp 611-616

10. Canudas-de-wit C, Tsiotras P, Velenis E, Basset M, Gissinger G (2002) Dynamic friction models for road/tire longitudinal interaction. Int J Veh Mech Mobil 39(3):189-226

11. Jansson J, Gustafsson F, Ekmark J (2002) Decision making for collision avoidance systems. In: SAE International, Warrendale, PA. SAE Technical Paper 2002-01-0403

12. Lin J-S, Ting W (2007) Nonlinear control design of anti-lock braking systems with assistance of active suspension. IET Control Theory Appl 1(1):343-348 